\title{
EXACT ANALYTIC SOLUTIONS DESCRIBING UNSTEADY PLANE GAS FLOWS WITH SHOCKS OF ARBITRARY STRENGTH
}

\author{
BY \\ P. L. SACHDEV*, NEELAM GUPTA, AND D. S. AHLUWALIA \\ New Jersey Institute of Technology, Newark, New Jersey
}

\begin{abstract}
Several classes of exact analytic solutions for one-dimensional plane gasdynamic equations describing piston-driven shocks propagating into inhomogeneous media are presented. These solutions are obtained by writing the conservation forms of the basic equations, and hence using their equivalent differential forms. These forms enable introduction of special coordinate systems which make it possible to exactly satisfy boundary conditions at the shock and at the piston. Previous results of Sachdev and Reddy [1] and Ustinov [2,3] are recovered as special cases. Several representative solutions are shown graphically. Strong shocks as well as shocks of arbitrary strength and characteristic fronts are considered.
\end{abstract}

1. Introduction. In a previous paper (Sachdev and Reddy [1]) we gave a class of similarity solutions of systems of one-dimensional gas-dynamic equations, expressed in a form in which the independent variables are $\tau$ and $s$. The level lines $\tau=$ constant are particle paths while $s=0$ demarcates the shock locus (Ustinov [2]). This coordinate system assisted in the satisfaction of boundary conditions both at the shock and the piston, and proved a useful vehicle for generating a large class of solutions which are not self-similar in the physical plane. Thus, the restriction of self-similarity in the physical plane was removed. The use of invariance property for the governing PDE ensured the inclusion of all the self-similar forms of solutions in the $(\tau, s)$-plane. These solutions describe piston-driven shocks in inhomogeneous media. The method is an inverse one: the solution is determined by the PDE and the Rankine-Hugoniot relations with given ambient conditions ahead; the piston motion in a parametric form generating the shock wave is then found simply by putting $\tau=0$ in the solution previously determined. Ustinov's solution [2] was recovered as a special case.

We generalize the study of Sachdev et al. in the following important ways:

I. The system of PDE (Eqs. (3.10)-(3.11)) in $(\tau, s)$-coordinates is analyzed more completely by dispensing with the restriction of self-similarity. The new class of solutions is found as analytic series in variables $W$ and $H$ (see $(4.7)-(4.8))$. The

Received October 24, 1990.

* Current address: Department of Mathematics, Indian Institute of Science, Bangalore, India.

(C)1992 Brown University 
similarity solutions of Sachdev and Reddy are identified as special cases of the more general series solutions obtained here.

II. The $(\tau, s)$-coordinates of Ustinov are replaced by another $(\tau, s)$ system which arises from the conservation of mass and energy; Ustinov's form comes from conservation of mass and momentum. With this new formulation, the type of solutions detailed in I above, including self-similar ones, are derived.

III. Strong shock solutions are derived for both the coordinate systems referred to in I and II. Some other solutions of Ustinov [3] for strong shocks are rederived.

IV. The class of solutions with characteristic boundaries rather than shocks is considerably enlarged.

An interesting feature of all the solutions found in the form of Taylor series of the variables $W$ and $H$ about $s=0$ is that the recurrence relations for the unknown coefficient functions in the series are not ODE but algebraic relations involving the previous coefficients and their derivatives. Thus, no ODE need be solved. This feature results from the special structures of the PDEs which do not involve $s$ explicitly in the derivative terms.

As a result of the above generalizations, we now have access to a very large class of solutions, not found hitherto. Their physical nature is rather similar to that found by Sachdev and Reddy. Major classes of solutions may be typified by the relations between particle velocity $u$, (essentially) pressure $H$, and either time $t$ or distance $x$ or both $x$ and $t$. These solutions are special simple forms, representative of the more general solutions, and constitute intermediate integrals. We summarize the main physical types as follows:

1. $u=\frac{p_{0} t_{0}}{\rho_{0} x_{0}}\left(1-\frac{a x}{x_{0}}\right)^{2} \exp \left(\frac{a t}{t_{0}}\right)\left[H^{i /\left(\eta^{\prime}-1\right)}-1\right]$.

2. $u=\frac{p_{0} t_{0}}{\rho_{0} x_{0}} \exp \left(-\frac{a x}{x_{0}}\right)\left[1+\frac{a t}{2 t_{0}}\right]$

$$
\cdot\left[\left(1+\frac{\gamma+1}{\gamma-1} H_{*}^{i /(\gamma-1)}+2 b_{0} H^{i /(\gamma-1)}-\frac{2 \gamma^{2} c_{*}}{\gamma-1} H\right)^{1 / 2}-1\right]^{1 / 2} \text {. }
$$

3. $u=\left[\frac{2}{\rho_{0}} \exp \left(-\frac{a x}{x_{0}}\right)\left\{\left(W^{(0)}+a_{0} \gamma H+a_{1}\right)\left(\frac{H}{H_{*}}\right)^{\gamma /\left(\gamma^{\prime}-1\right)}-\gamma a_{0} H-a_{1}\right.\right.$

$$
\left.\left.+(\gamma-1) a_{0} H^{\gamma^{\prime /(\gamma-1)}} /(H(0))^{1 /(\gamma-1)}\right\}\right]^{1 / 2} .
$$

4. $u=\frac{x_{0}}{t_{0}} \exp \left(-\frac{a t}{t-0}\right) H^{i /(;-1)}$.

5. $u=\frac{x_{0}}{t_{0}}\left(1+\frac{a t}{2 t_{0}}\right)^{-1}\left[2\left\{H^{i /(\gamma-1)}-\gamma c H\right\}\right]^{1 / 2}$.

6. $u=\frac{p_{0} t_{0}}{\rho_{0} x_{0}} \exp \left(-\frac{a x}{x_{0}}\right)\left[1+\frac{a t}{2 t_{0}}\right]\left[\left\{2 \gamma\left(H^{\gamma /(\gamma-1)}+\frac{1}{\gamma-1}(1-\gamma H)\right)\right\}^{1 / 2}-1\right]^{1 / 2}$. 
We believe that the extensive studies on the transformations of gas-dynamic equations carried out by Steketee $[4,5]$ would in the future be exploited to discover the solutions satisfying other boundary conditions.

This paper is organized as follows. Section 2 gives the basic equations and boundary conditions. These are transformed in Sec. 3 to the $(\tau, s)$-plane using the differential relations enunciating (a) conservation of mass and momentum, and (b) conservation of mass and energy. Solutions with shocks of finite strength for case (a) are found in Sec. 4, and those for case (b) are found in Sec. 5; the solutions of Sachdev and Reddy [1] are also recovered in Sec. 4. The strong shock case corresponding to (a) is discussed in Sec. 6, while that for (b) is considered in Sec. 7. The more general series solution for Ustinov's impulsive piston motion [3] is discussed in Sec. 6. The solutions with characteristic front are detailed in Sec. 8. The conclusions of the present study are set out in Sec. 9.

2. Basic equations and boundary conditions. The gas-dynamic equations governing one-dimensional (plane) unsteady anisentropic flows are

$$
\begin{gathered}
\rho_{t}+u \rho_{x}+\rho u_{x}=0, \\
\rho\left(u_{t}+u u_{x}\right)+p_{x}=0, \\
S_{t}+u S_{x}=0, \\
p=\rho^{\gamma} \exp \left(\frac{S-S_{0}}{c_{v}}\right),
\end{gathered}
$$

where $\rho, u, p$, and $S$ are the density of the fluid, particle velocity, pressure, and entropy, respectively, at any point $x$ and time $t$. The thermodynamic variables $p, \rho$, and $S$ are related by Eq. (2.4); $\gamma$ and $c_{v}$ denote the ratio of specific heats and specific heat at constant volume, respectively.

The system (2.1)-(2.3) may be written in conservation form as

$$
\begin{gathered}
\rho_{t}+(\rho u)_{x}=0 \\
(\rho u)_{t}+\left(\rho u^{2}+p\right)_{x}=0 \\
\left(\frac{1}{2} \rho u^{2}+\frac{p}{\gamma-1}\right)_{t}+\left(\left(\frac{1}{2} \rho u^{2}+\frac{p}{\gamma-1}\right) u+p u\right)_{x}=0 .
\end{gathered}
$$

The differential relations resulting from $(2.5)-(2.7)$ are the basis of transformations used in this paper to study the flows described by piston-driven shocks into nonuniform media. The Rankine-Hugoniot conditions holding across a shock moving into a nonuniform medium with velocity $u_{0}=0$, pressure $p=p_{0}$ (a constant), and density $\rho=\rho_{*}(x)$ are

$$
\begin{gathered}
u=\left(\frac{2}{\rho_{*}}\right)^{1 / 2}\left(p-p_{0}\right)[(\gamma+1) p+(\gamma+1)]^{-1 / 2}, \\
\frac{\rho}{\rho_{*}}=\frac{(\gamma+1) p+(\gamma-1) p_{0}}{(\gamma-1) p+(\gamma+1) p_{0}} \\
U=\left(2 \rho_{*}\right)^{-1 / 2}\left[(\gamma+1) p+(\gamma-1) p_{0}\right]^{1 / 2}
\end{gathered}
$$


where $U$ is the shock velocity; along the shock trajectory, $x=x(t)$, we have

$$
\frac{d x}{d t}=U
$$

For strong shocks Eqs. (2.8)-(2.10) reduce to

$$
\begin{gathered}
u=\left(\frac{2}{\gamma+1} \frac{p}{\rho_{*}}\right)^{1 / 2}, \\
\frac{\rho}{\rho_{*}}=\frac{\gamma+1}{\gamma-1}, \\
U=\left(\frac{\gamma+1}{2} \frac{p}{\rho_{*}}\right)^{1 / 2} .
\end{gathered}
$$

3. Transformations. Shocks of arbitrary strength.

A1. Equations (2.5) and (2.6) suggest the introduction of the variables $\tau$ and $\xi$ according to

$$
\begin{gathered}
d \tau=\rho d x-(\rho u) d t, \\
d \xi=\rho u d x-\left(p+\rho u^{2}\right) d t .
\end{gathered}
$$

These differential relations yield

$$
\begin{gathered}
d x=-\frac{u}{p} d \xi+\frac{\left(p+\rho u^{2}\right)}{\rho p} d \tau, \\
d t=-\frac{1}{p} d \xi+\frac{u}{p} d \tau .
\end{gathered}
$$

The shock trajectory (2.11) in terms of variables $\tau$ and $\xi$ becomes

$$
d \xi+\phi(\tau) d \tau=0
$$

where

$$
\phi(\tau)=\frac{p_{0}}{\rho_{*}(x)} U^{-1}
$$

To facilitate the fitting of the shock conditions $(2.8)-(2.10)$, a new variable $s$ is introduced such that

$$
d s=d \xi+\phi(\tau) d \tau
$$

which, in view of (3.5), ensures that $s$ remains constant along the shock. The differential relations (3.3)-(3.4) now become

$$
\begin{gathered}
d x=-\frac{u}{p} d s+\frac{\left(p+\rho u^{2}+\rho u \phi\right)}{\rho p} d \tau, \\
d t=-\frac{1}{p} d s+\frac{u+\phi}{p} d \tau .
\end{gathered}
$$

We may choose $s=0$ along a shock trajectory, since Eq. (3.7) is invariant under a translation in $s$. Equation (3.1) implies that $\tau=$ constant along a particle line. Thus, the trajectories $\tau=$ constant also give lines with constant entropy in the $(\tau, s)$ plane. In consequence of this, Eq. (2.3) may be dropped and the other two equations (2.1)-(2.2) may be transformed as

$$
u_{\tau}+(u+\phi) u_{s}-(f / \gamma) p^{-1 / \gamma} p_{s}=0
$$




$$
p_{\tau}+(u+\phi) p_{s}-p u_{s}=0,
$$

where

$$
f(\tau)=\left[p \rho^{-\gamma}\right]^{1 / \gamma}
$$

from the equation of state (2.4). We deal with the system (3.10)-(3.11) in Sec. 4.

A2. In view of (2.5) and (2.7), we introduce the variable $\tau$ as in (3.1), and the variable $\xi$ :

$$
d \xi=\left(\frac{1}{2} \rho u^{2}+\frac{p}{\gamma-1}\right) d x-\left(\left(\frac{1}{2} \rho u^{2}+\frac{p}{\gamma-1}\right) u+p u\right) d t .
$$

Equations (3.1) and (3.13) then give

$$
\begin{gathered}
d x=-\frac{1}{p} d \xi+\left(\left(\frac{1}{2} \rho u^{2}+\frac{p}{\gamma-1}\right)+p\right) \frac{1}{\rho p} d \tau, \\
d t=-\frac{1}{p u} d \xi+\left(\frac{1}{2} \rho u^{2}+\frac{p}{\gamma-1}\right) \frac{1}{\rho u p} d \tau .
\end{gathered}
$$

The shock trajectory $d x=U d t$ now becomes

$$
d \xi+\phi(\tau) d \tau=0
$$

where

$$
\phi(\tau)=-\frac{1}{\gamma-1} \frac{p_{0}}{\rho_{*}} .
$$

Again, we introduce a new variable $s$ such that

$$
d s=d \xi+\phi(\tau) d \tau
$$

implying $s=$ constant along the shock. Equations (3.14)-(3.15) may be replaced by

$$
\begin{aligned}
& d x=-\frac{d s}{p}+\left(\frac{1}{2} \rho u^{2}+\frac{p}{\gamma-1}+p+\rho \phi\right) \frac{1}{\rho p} d \tau, \\
& d t=-\frac{1}{p u} d s+\left(\frac{1}{2} \rho u^{2}+\frac{p}{\gamma-1}+\rho \phi\right) \frac{1}{\rho u p} d \tau .
\end{aligned}
$$

We assume that $s=0$ along a shock trajectory and $\tau=0$ along a piston path; $\tau=$ constant on any particle line.

For now Eq. (2.3) drops out and Eqs. (2.1)-(2.2) become

$$
\begin{gathered}
u_{\tau}+\left(\frac{1}{2} u^{2}+\frac{f p^{(\gamma-1) / \gamma}}{\gamma-1}+\phi\right) u_{s}-\frac{f}{\gamma} p^{-1 / \gamma} u p_{s}=0, \\
p_{\tau}+\left(\frac{1}{2} u^{2}+\frac{f p^{(\gamma-1) / \gamma}}{\gamma-1}+\phi\right) p_{s}-p u u_{s}=0,
\end{gathered}
$$

where $f$ is the same as in (3.12).

We consider the system (3.20)-(3.21) with boundary conditions $(2.8)-(2.10)$ at the shock in Sec. 5.

Strong shocks.

B1. It is easily found that the trajectory $(2.11)$ for a strong shock in the $(s, \tau)$ plane becomes

$$
d s=d \xi=0,
$$


where $s$ is the same as $\xi$ in (3.2). This may also be noted from (3.5), since $\phi$ in (3.6) tends to zero as $p_{0} \rightarrow 0$. Thus, $s=\xi=0$ gives the shock locus. When $\tau$ and $s$ from (3.1) and (3.2) are introduced as independent variables, Eqs. (2.1)-(2.3) take the form

$$
\begin{gathered}
u_{\tau}+u u_{s}-\frac{\gamma-1}{\gamma} f p^{-1 / \gamma} p_{s}=0, \\
p_{\tau}+u p_{s}-p u_{s}=0
\end{gathered}
$$

where

$$
f(\tau)=\frac{1}{\rho(\gamma-1)} p^{1 / \gamma} .
$$

The system (3.23)-(3.24) with shock boundary conditions $(2.12)-(2.14)$ will be considered in Sec. 6.

B2. The strong shock conditions $(2.12)-(2.14)$ together with the differential relation (3.13) suggest that the shock trajectory (2.11) may also be rewritten as

$$
d s=d \xi=0,
$$

where $s(=\xi)$ is given by (3.13). Thus, along the shock we have $s=0$.

Introduction of the variables $\tau$ and $s$ from (3.1) and (3.13) in (2.1)-(2.3) leads to

$$
\begin{gathered}
u_{\tau}+\left(\frac{1}{2} u^{2}+f p^{(\gamma-1) / \gamma}\right) u_{s}-\frac{\gamma-1}{\gamma} f u p^{-1 / \gamma} u_{s}=0, \\
p_{\tau}+\left(\frac{1}{2} u^{2}+f p^{(\gamma-1) / \gamma}\right) p_{s}-p u u_{s}=0,
\end{gathered}
$$

where $f$ is given by (3.25). The system (3.27)-(3.28) with boundary conditions (2.12)-(2.14) is considered in Sec. 7.

It may be noted that the transformed systems for shocks of finite strength (see (3.10)-(3.11) and (3.20)-(3.21)) involve two arbitrary functions $\phi(\tau)$ and $f(\tau)$, while for strong shocks (see (3.23)-(3.24) and (3.27)-(3.28)) they involve only one arbitrary function $f(\tau)$.

4. Solutions for the system (3.10)-(3.11) with shocks of finite strength. We introduce an arbitrary function $z(\tau)$, which will be chosen subsequently, and $s$ as the independent variable, and rewrite Eqs. (3.10)-(3.11) as

$$
\begin{gathered}
\phi^{2} W W_{s}-\frac{\beta f}{\gamma-1} H_{2}+\left((W-1) \frac{d \phi}{d z}+\phi W_{z}\right) \frac{d z}{d \tau}=0, \\
\phi W H_{s}-\frac{\gamma-1}{\gamma} \phi H W_{s}+H_{z} \frac{d z}{d \tau}=0,
\end{gathered}
$$

where

$$
W=1+\frac{u}{\phi}, \quad H=\frac{p^{(\gamma-1) / \gamma}}{\beta}, \quad \beta=p_{0}^{(\gamma-1) / \gamma},
$$

and $\phi$ is defined according to (3.6). The shock conditions (2.8)-(2.10) now become

$$
W_{s=0}=\left(H_{s=0}\right)^{\gamma /(\gamma-1)}
$$


and

$$
H_{s=0}=\left[\frac{1}{\gamma+1}\left(\frac{2 p_{0}}{\rho_{*} \phi^{2}}-\gamma+1\right)\right]^{(\gamma-1) / \gamma},
$$

while

$$
f(\tau)=\left[\frac{(\gamma-1) p_{0}+2 \gamma \rho_{*} \phi^{2}}{(\gamma+1) p_{0}^{(\gamma-1) / \gamma} \rho_{*}}\right]\left(H_{s=0}\right)^{1 /(\gamma-1)}
$$

follows from (3.12). We seek the solution of (4.1)-(4.2) in the series form

$$
\begin{aligned}
& W(z, s)=\sum_{j=0}^{\infty} W^{(j)}(z) \frac{s^{j}}{j !}, \\
& H(z, s)=\sum_{j=0}^{\infty} H^{(j)} \frac{s^{j}}{j !} .
\end{aligned}
$$

Substituting (4.7)-(4.8) into (4.1)-(4.2) and equating the coefficients of different powers of $s$ to zero, we find that

$$
\begin{gathered}
W^{(1)}(z)=\left[\phi W^{(0)} H^{(1)}-\frac{d H^{(0)}}{d z} \cdot \frac{d z}{d \tau}\right] \frac{\gamma}{(\gamma-1) \phi H^{(0)}}, \\
H^{(1)}(z)=\left[\left(W^{(0)}-1\right) \frac{d \phi}{d z}+\phi \frac{d W^{(0)}}{d z}+\beta \phi W^{(0)} \frac{d H^{(0)}}{d z}\right] \frac{(\gamma-1) H^{(0)}}{D_{0}} \frac{d z}{d \tau},
\end{gathered}
$$

and

$$
\begin{aligned}
& W^{(n+1)}(z)= {\left[\phi W^{(0)} H^{(n+1)}+\frac{d H^{(n)}}{d z} \frac{d z}{d \tau}+\phi T^{(n)}\left(\left\{W^{(i)}\right\},\left\{H^{(i)}\right\}\right)\right] } \\
& \cdot \frac{\gamma}{(\gamma-1) \phi H^{(0)}}, \\
& H^{(n+1)}(z)=\left[\left\{\gamma \phi W^{(0)} \frac{d H^{(n)}}{d z}+(\gamma-1) H^{(0)}\left(W^{(n)} \frac{d \phi}{d z}+\phi \frac{d W^{(n)}}{d z}\right)\right\} \frac{d z}{d \tau}\right. \\
&\left.+\gamma \phi^{2} W^{(0)} S^{(n)}\left(\left\{W^{(i)}\right\},\left\{H^{(i)}\right\}\right)\right]\left(D_{0}\right)^{-1}, \quad n=1,2, \ldots,
\end{aligned}
$$

where

$$
\begin{gathered}
T^{(n)}\left(\left\{W^{(i)}\right\},\left\{H^{(i)}\right\}\right)=\sum_{k=1}^{n}\left(\begin{array}{l}
n \\
k
\end{array}\right)\left[W^{(k)} H^{(n+1-k)}-\frac{\gamma-1}{\gamma} H^{(k)} W^{(n+1-k)}\right], \\
S^{(n)}\left(\left\{W^{(i)}\right\},\left\{H^{(i)}\right\}\right)=\sum_{k=1}^{n}\left(\begin{array}{l}
n \\
k
\end{array}\right)\left[W^{(k)} H^{(n+1-k)}+\frac{\gamma-1}{\gamma}\left(\frac{H^{(0)}}{W^{(0)}} W^{(k)}-H^{(k)}\right) W^{(n+1-k)}\right], \\
D_{0}=\beta f H^{(0)}-\gamma \phi^{2} W^{(0)^{2}} .
\end{gathered}
$$

The system of PDE (4.1)-(4.2) yields algebraic relations (instead of ODE) for $W^{(1)}$ 
and $H^{(1)}$, and hence for $W^{(j)}, H^{(j)}, j \geq 2$, recursively. This is so because the coefficients of the derivative terms in (4.1)-(4.2) do not depend explicitly on $s$.

To satisfy the boundary conditions $(4.4)-(4.6)$ at the shock, we may assume that $W^{(0)}$ and $H^{(0)}$ are given by $(4.4)$ and $(4.5)$, respectively. It is clear from these equations that if we choose $\rho_{*}, \phi$, and $f$ at the shock such that $\rho_{*} \phi^{2}$ and $f \rho_{*}$ are constant, then $W^{(0)}$ and $H^{(0)}$ would be constant. With constant basic solution, the algebraic relations for first-order coefficients simply become

$$
\begin{gathered}
W^{(1)}(z)=\frac{\gamma W^{(0)}}{(\gamma-1) H^{(0)}} H^{(1)}, \\
H^{(1)}(z)=\frac{(\gamma-1)\left(W^{(0)}-1\right) H^{(0)}}{D_{0}} \frac{d \phi}{d z} \cdot \frac{d z}{d \tau} .
\end{gathered}
$$

We first recover the results of Sachdev and Reddy [1] as well as those of Ustinov [2] as special cases of the more general series solution (4.7)-(4.8), with (4.4)-(4.5), $(4.14)-(4.15)$, and (4.11)-(4.13). The functions $z(\tau), \phi(\tau)$, etc., will be suitably chosen to yield these results.

(I) Power-law similarity solutions of the system (4.1)-(4.2). To recover the powerlaw similarity solutions of Sachdev and Reddy [1], we choose

$$
\begin{gathered}
z= \begin{cases}{\left[c_{0}\left(1+c_{1} \tau\right)\right]^{-\alpha},} & \alpha \neq-1, \\
c_{0}, & \alpha=-1,\end{cases} \\
\phi(z)=\frac{c_{2}}{z}, \\
f(z)=\frac{c_{3}}{z^{2}}, \\
\rho_{*}(z)=\rho_{0}\left(1+c_{1} \tau\right)^{-2 \alpha},
\end{gathered}
$$

where the constants $c_{0}, c_{1}, c_{2}$, and $c_{3}$ are defined as

$$
\begin{gathered}
c_{0}= \begin{cases}\left(p_{0} t_{0}\right)^{1 /(\alpha+1)}, & \alpha \neq-1, \\
1 /\left(p_{0} t_{0}\right), & \alpha=-1,\end{cases} \\
c_{1}=\frac{1}{\rho_{0} x_{0}}, \quad c_{2}=\left(\frac{p_{0} t_{0}}{\rho_{0} x_{0}}\right) c_{0}^{-\alpha}, \quad c_{3}=\frac{\gamma p_{0}^{(\gamma+1) / \gamma} c_{*} t_{0}^{2}}{\rho_{0} x_{0}^{2} c_{0}^{2 \alpha} .}
\end{gathered}
$$

In the above, $\alpha$ is the similarity exponent, and $x_{0}, t_{0}, p_{0}$, and $\rho_{0}$ are arbitrary constants with the dimensions of distance, time, pressure, and density, respectively. The constant $a$ assumes values +1 or -1 . The case $\alpha=0$ implies isentropy of the flow, and is not admitted by the basic transformation of Sec. 3 .

The parameter $c_{*}$ is given by

$$
c_{*}=\frac{b_{0}\left(\gamma-1+2 \gamma / b_{0}\right)}{\gamma(\gamma+1)} H_{*}^{1 /(\gamma-1)}
$$

where

$$
H_{*}=\left(\frac{2 b_{0}-\gamma+1}{\gamma+1}\right)^{(\gamma-1) / \gamma},
$$


and $b_{0}=\rho_{0} x_{0}^{2} /\left(p_{0} t_{0}^{2}\right)$. In view of (4.16)-(4.19), the basic solution is given by

$$
\begin{gathered}
W^{(0)}=\frac{2 b_{0}-\gamma+1}{\gamma+1}, \\
H^{(0)}=H_{*}=\left[\frac{2 b_{0}-\gamma+1}{\gamma+1}\right]^{(\gamma-1) / \gamma}
\end{gathered}
$$

(see (4.4)-(4.5)). Using (4.16)-(4.23) in (4.14)-(4.15), we have

$$
\begin{aligned}
& W^{(1)}(z)= \begin{cases}W_{*}^{(1)} z^{(\alpha+1) / \alpha}, & \alpha \neq-1, \\
W_{*}^{(1)}\left(1 / p_{0} t_{0}\right), & \alpha=-1,\end{cases} \\
& H^{(1)}(z)= \begin{cases}H_{*}^{(1)} z^{(\alpha+1) / \alpha}, & \alpha \neq-1, \\
H_{*}^{(1)}\left(1 / p_{0} t_{0}\right), & \alpha=-1,\end{cases}
\end{aligned}
$$

where constants $W_{*}^{(1)}$ and $H_{*}^{(1)}$ are given by

$$
\begin{aligned}
& W_{*}^{(1)}=\frac{\gamma W^{(0)}}{(\gamma-1) H^{(0)}} H_{*}^{(1)}, \\
& H_{*}^{(1)}=\frac{\gamma-U\left(1-W^{(0)}\right) a \alpha}{\gamma D_{*}} H^{(0)}, \quad D_{*}=W^{(0)^{2}}-c_{*} H^{(0)} .
\end{aligned}
$$

Again, using (4.16)-(4.23) in (4.11)-(4.13), we find that the second and higher order coefficients, $W^{(j)}(z)$ and $H^{(j)}(z), j=n+1$, with $n=1,2, \ldots$, in the series (4.7)-(4.8) have the form

$$
\begin{aligned}
& W^{(n+1)}(z)= \begin{cases}W_{*}^{(n+1)} z^{(n+1)(\alpha+1) / \alpha}, & \alpha \neq-1, \\
W_{*}^{(n+1)}\left(1 / p_{0} t_{0}\right)^{n+1}, & \alpha=-1,\end{cases} \\
& H^{(n+1)}(z)= \begin{cases}H_{*}^{(n+1)} z^{(n+1)(\alpha+1) / \alpha}, & \alpha \neq-1, \\
H_{*}^{(n+1)}\left(1 / p_{0} t_{0}\right)^{n+1}, & \alpha=-1,\end{cases}
\end{aligned}
$$

where

$$
\begin{gathered}
W_{*}^{(n+1)}=\frac{1}{H^{(0)}}\left[\frac{\gamma}{\gamma-1} W^{(0)} H_{*}^{(n+1)}-\frac{a n \gamma(\alpha+1)}{(\gamma-1)} H_{*}^{(n)}\right. \\
\left.\quad-\sum_{k=1}^{n}\left(\begin{array}{l}
n \\
k
\end{array}\right)\left(H_{*}^{(k)} W_{*}^{(n=1-k)}-\frac{\gamma}{\gamma-1} W_{*}^{(k)} H_{*}^{(n+1-k)}\right)\right], \\
\begin{aligned}
H_{*}^{(n+1)}=\frac{(\gamma-1) H^{(0)}}{\gamma D_{*}}\left[\begin{array}{l}
\frac{a n \gamma(\alpha+1) W^{(0)}}{(\gamma-1) H^{(0)}} H_{*}^{(n)}+(n a(\alpha+1)-a \alpha) W_{*}^{(n)} \\
+\sum_{k=1}^{n}\left(\begin{array}{l}
n \\
k
\end{array}\right)\left(\left(\frac{W^{(0)}}{H^{(0)}} H_{*}^{(k)}-W_{*}^{(k)}\right)\right.
\end{array}\right) W_{*}^{(n+1-k)} \\
\left.\left.\frac{-\gamma W^{(0)}}{(\gamma-1) H^{(0)}} W_{*}^{(n)} H_{*}^{(n+1-k)}\right)\right]
\end{aligned}
\end{gathered}
$$

are constants. This may be proved by an induction argument. 
In view of the form $(4.24)-(4.25),(4.27)-(4.28)$ of the coefficient functions, the series $(4.7)-(4.8)$, in this case, can be rewritten as

$$
\begin{aligned}
& W=\sum_{j=0}^{\infty} W_{*}^{(j)} \frac{\sigma^{j}}{j}, \\
& H=\sum_{j=0}^{\infty} H_{*}^{(j)} \frac{\sigma^{j}}{j !},
\end{aligned}
$$

where

or

$$
\sigma= \begin{cases}s z^{(\alpha+1) / \alpha}, & \alpha \neq-1 \\ s / p_{0} t_{0}, & \alpha=-1\end{cases}
$$

$$
\sigma=\frac{s}{p_{0} t_{0}}\left(1+\frac{a \tau}{\rho_{0} x_{0}}\right)^{-(\alpha+1)}
$$

and $W_{*}^{(0)}=W^{(0)}$ and $H_{*}^{(0)}=H^{(0)}$. We note that the series solution $(4.31)-(4.32)$, (4.22)-(4.23), (4.26), and (4.29)-(4.30) is an exact solution of the following ODE, with boundary conditions $(4.22)-(4.23)$ at the shock:

$$
\begin{gathered}
(W-a(\alpha+1) \sigma) \frac{d W}{d \sigma}-c_{*} p^{-1 / \gamma} \frac{d p}{d \sigma}=a \alpha(1-W), \\
p \frac{d W}{d \sigma}-(W-\alpha(\alpha+1) \sigma) \frac{d p}{d \sigma}=0,
\end{gathered}
$$

with $\sigma$ as in (4.33), and $p=H^{\gamma /(\gamma-1)}$. These ODE describe power-law similarity solutions of Sachdev and Reddy [1].

Along the shock, Eq. (3.8) gives

$$
d \tau=\rho_{*} d x
$$

Substituting $\rho_{*}$ from (4.19) into (4.36) and integrating, we get

$$
\frac{x}{x_{0}}= \begin{cases}\frac{1}{a(2 \alpha+1)}\left[\left(1+\frac{a \tau}{\rho_{0} x_{0}}\right)^{2 \alpha+1}-1\right], & \alpha \neq-\frac{1}{2}, \\ \frac{1}{a} \ln \left(1+\frac{a \tau}{\rho_{0} x_{0}}\right), & \alpha=-\frac{1}{2} .\end{cases}
$$

Again, using $d x=U d t$ in (4.36), we get, after an integration,

$$
\frac{t}{t_{0}}= \begin{cases}\frac{1}{a(\alpha+1)}\left[\left(1+\frac{a \tau}{\rho_{0} x_{0}}\right)^{\alpha+1}-1\right], & \alpha \neq-1, \\ \frac{1}{a} \ln \left(1+\frac{a \tau}{\rho_{0} x_{0}}\right), & \alpha=-1 .\end{cases}
$$

In obtaining (4.37) and (4.38), we have used the boundary conditions $\tau=0$ at $x=0, t=0$. Equations (4.37)-(4.38) give the parametric representation of the shock trajectory. Eliminating $\tau$ between these equations, we get

$$
\frac{x(t)}{x_{0}}= \begin{cases}\frac{1}{a(2 \alpha+1)}\left[\left(1+\frac{a(\alpha+1) t}{t_{0}}\right)^{(2 \alpha+1) /(\alpha+1)}-1\right], & \alpha \neq-1,-\frac{1}{2}, \\ \frac{1}{a}\left[1-\exp \left(-\frac{a t}{t_{0}}\right)\right], & \alpha=-1, \\ \frac{2}{a} \ln \left(1+\frac{a t}{t_{0}}\right), & \alpha=-\frac{1}{2},\end{cases}
$$


the trajectory of the shock in the $(x, t)$-plane.

Expressing $\tau$ as a function of $x$ from (4.37) and substituting into (4.19), we get

$$
\rho_{*}(x)= \begin{cases}\rho_{0}\left[1+(2 \alpha+1) a \frac{x}{x_{0}}\right]^{-2 \alpha /(2 \alpha+1)}, & \alpha \neq-\frac{1}{2}, \\ \rho_{0} \exp \left(\frac{a x}{x_{0}}\right), & \alpha=-\frac{1}{2} .\end{cases}
$$

Similarly, substituting $\tau$ as a function of $t$ from (4.38) into the expression for the shock velocity, we obtain

$$
U(t)= \begin{cases}\frac{x_{0}}{t_{0}}\left(1+(\alpha+1) a \frac{t}{t_{0}}\right)^{\alpha /(\alpha+1)}, & \alpha \neq-1, \\ \frac{x_{0}}{t_{0}} \exp \left(-\frac{a t}{t_{0}}\right), & \alpha=-1 .\end{cases}
$$

Now the functions $x$ and $t$ may be found to be

$$
\frac{x(\tau, H)}{x_{0}}=\left\{\begin{array}{cc}
\frac{\left(1+a \tau / \rho_{0} x_{0}\right)^{2 \alpha+1}}{a b_{0} \alpha} \int_{H_{*}}^{H}\left[\frac{\gamma-1}{\gamma} H\left(\frac{d W}{d H}\right)^{2}-c_{*} \frac{\gamma}{\gamma-1}\right] H^{-\gamma /(\gamma-1)} d H \\
+\frac{1}{a(2 \alpha+1)}\left[\left(1+\frac{a \tau}{\rho_{0} x_{0}}\right)^{2 \alpha+1}-1\right], & \alpha \neq-\frac{1}{2}, \\
\frac{2}{a b_{0}} \int_{H_{*}}^{H}\left[\frac{\gamma}{\gamma-1} c_{*}-\frac{\gamma-1}{\gamma} H\left(\frac{d W}{d H}\right)^{2}\right] H^{-\gamma /(\gamma-1)} d H \\
+\frac{1}{a} \ln \left(1+\frac{a \tau}{\rho_{0} x_{0}}\right), & \alpha=-\frac{1}{2},
\end{array}\right.
$$

and

$$
\frac{t(\tau, H)}{t_{0}}=\left\{\begin{array}{cc}
\frac{\left(1+a \tau / \rho_{0} x_{0}\right)^{\alpha+1}}{a \alpha} \int_{H_{*}}^{H}\left[\frac{\gamma-1}{\gamma} H\left(\frac{d W}{d H}\right)^{2}-c_{*} \frac{\gamma}{\gamma-1}\right] \frac{H^{-\gamma /(\gamma-1)}}{(W-1)} d H \\
+\frac{1}{a(\alpha+1)}\left[\left(1+\frac{a \tau}{\rho_{0} x_{0}}\right)^{\alpha+1}-1\right], \quad \alpha \neq-1, \\
\frac{1}{a} \int_{H_{*}}^{H}\left[\frac{\gamma c_{*}}{\gamma-1}-\frac{\gamma-1}{\gamma} H\left(\frac{d W}{d H}\right)^{2}\right] \frac{H^{-\gamma /(\gamma-1)}}{(W-1)} d H \\
\frac{1}{a} \ln \left(1+\frac{a \tau}{\rho_{0} x_{0}}\right), & \alpha=-1,
\end{array}\right.
$$

from (3.8)-(3.9). Here

$$
\begin{gathered}
H_{*} \leq H<\infty \text { for } a \alpha>0, \\
1<H \leq H_{*} \text { for } a \alpha<0 .
\end{gathered}
$$

Setting $\tau=$ constant in (4.42) and (4.43), we obtain the trajectories of the gas particles in a parametric form. In particular, with $\tau=0$, we obtain the law of motion of the piston as

$$
\begin{aligned}
\frac{x(H)}{x_{0}} & =\frac{1}{a b_{0} \alpha} \int_{H_{*}}^{H}\left[\frac{\gamma-1}{\gamma} H\left(\frac{d W}{d H}\right)^{2}-c_{*} \frac{\gamma}{\gamma-1}\right] H^{-\gamma /(\gamma-1)} d H, \\
\frac{t(H)}{t_{0}} & =\frac{1}{a \alpha} \int_{H_{*}}^{H}\left[\frac{\gamma-1}{\gamma} H\left(\frac{d W}{d H}\right)^{2}-c_{*} \frac{\gamma}{\gamma-1}\right] \frac{H^{-\gamma /(\gamma-1)}}{(W-1)} d H .
\end{aligned}
$$


Thus, we have obtained an exact solution of the following problem: at $t=0$, a piston begins to move into a quiescent gas in $x>0$ with pressure equal to $p_{0}$ and density $\rho_{*}(x)$ given by (4.40). It follows the trajectory (4.44). Ahead of the piston a shock appears immediately which subsequently propagates according to (4.39). The solution (4.31)-(4.32), (4.22)-(4.23), (4.26), (4.29), and (4.30) of this problem involves five arbitrary (dimensional) constants: $x_{0}, t_{0}, \rho_{0}, p_{0}$, and $\alpha$.

The following points emerge. When $a \alpha>0 \quad(<0)$ and the undisturbed density, $\rho_{*}$, is a decreasing (increasing) function of $x$, the shock velocity increases (decreases) with $t$, the nondimensional pressure at the back of the shock $P=H^{\gamma /(\gamma-1)} \geq H_{*}^{\gamma /(\gamma-1)}$ $\left(1<P \leq H_{*}^{\gamma /(\gamma-1)}\right)$. The detailed dependence of the shock velocity on the undisturbed density is as follows. If $0>\alpha>-1 / 2$ and $a>0 \quad(<0)$ in (4.40)-(4.41), $\rho_{*}$ grows to infinity in an infinite distance and shock velocity decays to zero in an infinite time $\left(\rho_{*}\right.$ decays to zero in a finite distance $x=\left|x_{0} / a(2 \alpha+1)\right|$, and $U$ grows to infinity in a finite time $\left.t=\left|t_{0} / a(\alpha+1)\right|\right)$. For $-1 / 2>\alpha>-1$ and $a>0 \quad(<0)$, $\rho_{*}$ grows to infinity in finite distance, $x=\left|x_{0} / a(2 \alpha+1)\right|$, while $U$ vanishes in an infinite time $\left(\rho_{*}\right.$ becomes zero in an infinite distance and $U$ becomes infinite in finite time $\left.t=\left|t_{0} / a(\alpha+1)\right|\right)$. For $-1>\alpha>-\infty$ and $a>0 \quad(<0), \rho_{*}$ becomes infinite in a finite distance $x=\left|x_{0} / a(2 \alpha+1)\right|$ and shock velocity vanishes in finite time $t=\left|t_{0} / a(\alpha+1)\right| \quad\left(\rho_{*}\right.$ vanishes and $U$ grows to infinity in an infinite distance and infinite time, respectively). For $\alpha=-1 / 2$ and $a>0 \quad(<0), \rho_{*}$ grows exponentially and $U$ decays to zero in an infinite distance and infinite time, respectively ( $\rho_{*}$ decays exponentially to assume zero value in an infinite distance, while $U$ grows to infinity in a finite time $\left.t=2 t_{0}\right)$. For $\alpha=-1$ and $a>0 \quad(<0), \rho_{*}$ becomes infinite in a finite distance $x=x_{0}$, while $U$ decays exponentially in an infinite time ( $\rho_{*}$ vanishes in an infinite distance and $U$ grows exponentially in an infinite time).

If we denote the ratio of the shock velocity to the sound speed in the quiescent gas by $M_{0}$, then $M_{0}=\left(b_{0} / \gamma\right)^{1 / 2}$. In view of the second law of thermodynamics $M_{0}>1$; that is, $b_{0}>\gamma$.

We note that the series (4.31)-(4.32), using (4.22)-(4.23), (4.26), and (4.29)(4.30), can be summed up for $\alpha=-1,-1 / 2$. In fact, the ODE (4.34)-(4.35) could be solved in closed form in [1] only for these value of $\alpha$.

For $\alpha=-1$, Eqs. (4.26) give

$$
\begin{gathered}
W_{*}^{(1)}=\frac{\gamma W^{(0)}}{(\gamma-1) H^{(0)}} H_{*}^{(1)}, \\
H_{*}^{(1)}=\frac{(\gamma-1)\left(W^{(0)}-1\right)}{\gamma D_{*}} H^{(0)},
\end{gathered}
$$

where

$$
W^{(0)}=\left(H^{(0)}\right)^{i /(;-1)}
$$


from (4.22)-(4.23). That is,

$$
W_{*}^{(1)}=\frac{\gamma}{\gamma-1}\left(H^{(0)}\right)^{1 /(\gamma-1)} H_{*}^{(1)} .
$$

Similarly, Eqs. (4.29)-(4.30) give

$$
\begin{gathered}
W_{*}^{(2)}=\frac{2 \gamma}{\gamma-1}\left(H^{(0)}\right)^{1 /(\gamma-1)} H_{*}^{(2)}+\frac{\gamma}{(\gamma-1)^{2}}\left(H^{(0)}\right)^{(2-\gamma) /(\gamma-1)}\left(H_{*}^{(1)}\right)^{2}, \\
W_{*}^{(3)}=\frac{\gamma}{\gamma-1}\left(H^{(0)}\right)^{1 /(\gamma-1)} H_{*}^{(3)} \\
\quad=\frac{3 \gamma}{(\gamma-1)^{2}}\left(H^{(0)}\right)^{(2-\gamma) /(\gamma-1)} H_{*}^{(1)} H_{*}^{(2)}+\frac{\gamma(2-\gamma)}{(\gamma-1)^{3}}\left(H_{*}^{(1)}\right)^{3},
\end{gathered}
$$

etc., for $n=1,2, \ldots$. In view of $(4.47)-(4.50)$, the series $(4.31)-(4.32)$ can be summed up to give

$$
W=H^{\gamma /(\gamma-1)},
$$

since the right-hand sides of (4.47), (4.48), (4.49), and (4.50) are, respectively, zeroth, first, second, and third order derivative terms in the Taylor expansion of $H^{\gamma /(\gamma-1)}$ about $\sigma=0$. Similarly, the higher order coefficients can also be found. Thus, the solution for $\alpha=-1$ can be written in the following form:

$$
\begin{aligned}
& u=\phi\left(H^{\gamma /(\gamma-1)}-1\right), \\
& \phi=\frac{p_{0} t_{0}}{\rho_{0} x_{0}}\left(1-\frac{a x}{x_{0}}\right)^{2} \exp \left(\frac{a t}{t_{0}}\right) \text {, } \\
& f(\tau)=\frac{\gamma p_{0}^{1 / \gamma}}{b_{0} \rho_{0}} c_{*}\left(1+\frac{a \tau}{\rho_{0} x_{0}}\right)^{-2} \\
& \rho_{*}=\rho_{0}\left(1-\frac{a x}{x_{0}}\right)^{-2} \\
& \frac{x(t)}{x_{0}}=\frac{1}{a}\left[1-\exp \left(-\frac{a t}{t_{0}}\right)\right] \text {, } \\
& \frac{x(H, \tau)}{x_{0}}=\frac{\gamma\left(1+a \tau / \rho_{0} x_{0}\right)^{-1}}{(\gamma-1) a b_{0}}\left[\gamma c_{*}\left(H_{*}^{-1 /(\gamma-1)}-H^{-1 /(\gamma-1)}\right)-\left(H^{\gamma /(\gamma-1)}-H_{*}^{\gamma /(\gamma-1)}\right)\right] \\
& +\frac{1}{a}\left[1-\left(1+\frac{a \tau}{\rho_{0} x_{0}}\right)^{-1}\right] \text {, } \\
& \frac{t(H, \tau)}{t_{0}}=\frac{\gamma}{a(\gamma-1)} \int_{H_{*}}^{H} \frac{\left(c_{*}-H^{(\gamma+1) /(\gamma-1)}\right)}{\left(H^{\gamma /(\gamma-1)}-1\right)} H^{-\gamma^{\prime /(\gamma-1)}} d H+\frac{1}{a} \ln \left(1+\frac{a \tau}{\rho_{0} x_{0}}\right),
\end{aligned}
$$

$1<H \leq H_{*}$ for $a=1$, and $H_{*} \leq H<\infty$ for $a=-1$ (cf. [1]). 
Setting $a=-1$ in (4.52), we have

$$
\begin{gathered}
u=\phi\left(H^{\gamma /(\gamma-1)}-1\right), \quad \phi=\frac{p_{0} t_{0}}{\rho_{0} x_{0}}\left(1+\frac{x}{x_{0}}\right)^{2} \exp \left(-\frac{t}{t_{0}}\right), \\
f(\tau)=\frac{\gamma p_{0}^{1 / \gamma}}{b_{0} \rho_{0}} c_{*}\left(1-\frac{\tau}{\rho_{0} x_{0}}\right)^{-2}, \quad \rho_{*}=\rho_{0}\left(1+\frac{x}{x_{0}}\right)^{-2}, \\
\frac{x(t)}{x_{0}}=\frac{1}{a}\left[1-\exp \left(\frac{t}{t_{0}}\right)\right] \\
\frac{x(H, \tau)}{x_{0}}=\frac{\gamma\left(1-\tau / \rho_{0} x_{0}\right)^{-1}}{(\gamma-1) b_{0}}\left[\left(H^{\gamma /(\gamma-1)}-H_{*}^{-\gamma /(\gamma-1)}\right)-\gamma c_{*}\left(H_{*}^{-1 /(\gamma-1)}-H^{-1 /(\gamma-1)}\right)\right] \\
+\left[\left(1-\frac{\tau}{\rho_{0} x_{0}}\right)^{-1}\right], \\
\frac{t(H, \tau)}{t_{0}}=\frac{\gamma}{(\gamma-1)} \int_{H_{*}}^{H}\left(H^{(\gamma+1) /(\gamma-1)}-c_{*}\right) \frac{H^{-\gamma /(\gamma-1)}}{\left(H^{\gamma /(\gamma-1)}-1\right)} d H-\ln \left(1-\frac{\tau}{\rho_{0} x_{0}}\right),
\end{gathered}
$$

with $H=\left(p / p_{0}\right)^{(\gamma-1) / \gamma}$ (cf. [2]).

For $\alpha=-1 / 2$, we assume that

$$
W=(G(H))^{1 / 2}+1 \quad \text { and } \quad G(H)=G^{(0)}+\sum_{i=1}^{\infty}\left[\frac{d^{i} G}{d H^{i}}\right]_{H=H_{*}} \frac{\sigma^{i}}{i !}
$$

Now Eqs. (4.22)-(4.23), (4.26), and (4.29)-(4.30) give

$$
\begin{aligned}
G^{(0)} & =\left(1-H_{*}^{\gamma /(\gamma-1)}\right)^{2}, \\
\left(\frac{d G}{d H}\right)_{H=H_{*}} & =\frac{2 b_{0} \gamma}{\gamma-1} H_{*}^{1 /(\gamma-1)}-\frac{2 \gamma^{2} c_{*}}{\gamma-1}, \\
\left(\frac{d^{2} G}{d H^{2}}\right)_{H=H_{*}} & =\frac{2 b_{0} \gamma}{(\gamma-1)^{2}} H_{*}^{(2-\gamma) /(\gamma-1)}, \\
\left(\frac{d^{3} G}{d H^{3}}\right)_{H=H_{*}} & =\frac{2 b_{0} \gamma(2-\gamma)}{(\gamma-1)^{3}} H_{*}^{(3-2 \gamma) /(\gamma-1)},
\end{aligned}
$$

etc. The series for $G$ in (4.54) may now be summed up to give

$$
G=1+\frac{\gamma+1}{\gamma-1} H_{*}^{\gamma /(\gamma-1)}+2 b_{0} H^{\gamma /(\gamma-1)}-\frac{2 \gamma^{2} c_{*}}{\gamma-1} H .
$$


Again, the solution for $\alpha=-1 / 2$ may be written in a closed form as

$$
\begin{gathered}
u=\phi G^{1 / 2}, \quad \phi=\frac{p_{0} t_{0}}{\rho_{0} x_{0}} \exp \left(-\frac{a x}{x_{0}}\right)\left[1+\frac{a t}{2 t_{0}}\right] \\
f=\frac{\gamma p_{0}^{1 / \gamma} c_{*}}{b_{0} \rho_{0}}\left(1+\frac{a \tau}{\rho_{0} x_{0}}\right)^{-1}, \quad \rho_{*}=\rho_{0} \exp \left(\frac{a x}{x_{0}}\right) \\
\frac{x(t)}{x_{0}}=\frac{2}{a} \ln \left(1+\frac{a t}{t_{0}}\right), \\
\frac{x(H, \tau)}{x_{0}}=\frac{2}{a b_{0}} \int_{H_{*}}^{H}\left[\frac{\gamma}{\gamma-1} c_{*}-\frac{\gamma-1}{\gamma} H\left(\frac{d W}{d H}\right)^{2}\right] H^{-\gamma /(\gamma-1)} d H \\
\frac{t(H, \tau)}{t_{0}}=\frac{2\left(1+a \tau / \rho_{0} x_{0}\right)^{1 / 2}}{a} \int_{H_{*}}^{H}\left[\frac{a \tau}{\rho_{0} x_{0}}\right), 1 \\
+\frac{2}{a}\left[\left(1+\frac{a \tau}{\rho_{0} x_{0}}\right)^{1 / 2}-\frac{\gamma-1}{\gamma} H\left(\frac{d W}{d H}\right)^{2}\right] \frac{H_{*}^{-\gamma /(\gamma-1)}}{(W-1)} d H
\end{gathered}
$$

where $1<H \leq H_{*}$ for $a=1$, and $H_{*} \leq H<\infty$ for $a=-1 ; W$ is given by (4.54) and (4.59) (cf. [1]).

(II) Exponential similarity solutions for the system (4.1)-(4.2). We now choose

$$
\begin{aligned}
z & =\frac{1}{p_{0} t_{0}} \exp \left(-\frac{a \tau}{\rho_{0} x_{0}}\right), \\
\phi & =\frac{p_{0} t_{0}}{\rho_{0} x_{0}} \exp \left(\frac{a \tau}{\rho_{0} x_{0}}\right), \\
f & =\frac{\gamma p_{0}^{1 / \gamma}}{b_{0} \rho_{0}} c_{*} \exp \left(\frac{2 a \tau}{\rho_{0} x_{0}}\right), \\
\rho_{*} & =\rho_{0} \exp \left(-\frac{2 a \tau}{\rho_{0} x_{0}}\right),
\end{aligned}
$$

where $c_{*}$ is given by $(4.21), \rho_{0}, x_{0}, t_{0}$, and $p_{0}$ are (dimensional) constants, and $b_{0}=\rho_{0} x_{0}^{2} /\left(p_{0} t_{0}^{2}\right)$. The zeroth-order terms in the solution series $(4.7)-(4.8)$ are

$$
\begin{gathered}
W^{(0)}=\left(H^{0}\right)^{\gamma /(\gamma-1)}, \\
H^{(0)}=H_{*},
\end{gathered}
$$

where $H_{*}$ is defined in (4.23).

Substituting (4.61)-(4.64) into (4.14)-(4.15), we get

$$
\begin{aligned}
& W^{(1)}(z)=W_{*}^{(1)} z, \\
& H^{(1)}(z)=H_{*}^{(1)} z,
\end{aligned}
$$


where

$$
\begin{aligned}
& W_{*}^{(1)}=\frac{\gamma W^{(0)}}{(\gamma-1) H^{(0)}} H_{*}^{(1)}, \\
& H_{*}^{(1)}=\frac{(\gamma-1) a\left(1-W^{(0)}\right) H^{(0)}}{\gamma D_{*}},
\end{aligned}
$$

with

$$
D_{*}=W_{*}^{(0)^{2}}-c_{*} H_{*}, \quad W_{*}^{(0)}=W^{(0)}, \quad H_{*}^{(0)}=H^{(0)} .
$$

Using (4.61)-(4.70) in (4.11)-(4.13), the second and higher order coefficients $W^{(i)}$ and $H^{(i)}, i=n+1, n=1,2, \ldots$, in the series $(4.7)-(4.8)$ are found to be in the form

$$
\begin{aligned}
& W^{(n+1)}(z)=W_{*}^{(n+1)} z^{n+1}, \\
& H^{(n+1)}(z)=H_{*}^{(n+1)} z^{n+1},
\end{aligned}
$$

where

$$
\begin{aligned}
& W_{*}^{(n+1)}=\frac{1}{H_{*}^{(0)}}\left[\frac{\gamma}{\gamma-1}\left(W_{*}^{(0)} H_{*}^{(n+1)}-a n H_{*}^{(n)}\right)\right. \\
& \left.+\sum_{k=1}^{n}\left(\begin{array}{l}
n \\
k
\end{array}\right)\left(\frac{\gamma}{\gamma-1} W_{*}^{(k)} H_{*}^{(n+1-k)}-H_{*}^{(k)} W_{*}^{(n+1-k)}\right)\right], \\
& H_{*}^{(n+1)}=\frac{(\gamma-1) H_{*}^{(0)}}{\gamma D_{*}}\left[a(n-1) W_{*}^{(n)}+\frac{a n \gamma}{\gamma-1} \frac{W_{*}^{(0)}}{H_{*}^{(0)}} H_{*}^{(n)}\right. \\
& +\sum_{k=1}^{n}\left(\begin{array}{l}
n \\
k
\end{array}\right)\left(\left(\frac{W_{*}^{(0)}}{H_{*}^{(0)}} H_{*}^{(k)}-W_{*}^{(k)}\right) W_{*}^{(n+1-k)}\right. \\
& \left.\left.-\frac{\gamma}{\gamma-1} \frac{W_{*}^{(0)}}{H_{*}^{(0)}} W_{*}^{(k)} H_{*}^{(n+1-k)}\right)\right]
\end{aligned}
$$

are constants. Now the series solution (4.7)-(4.8) may be rewritten as

$$
\begin{aligned}
& W=\sum_{i=0}^{\infty} W_{*}^{(i)} \frac{\sigma^{i}}{i !}, \\
& H=\sum_{i=0}^{\infty} H_{*}^{(i)} \frac{\sigma^{i}}{i !}
\end{aligned}
$$

where $W_{*}^{(i)}$ and $H_{*}^{(i)}, i \geq 0$, are given by $(4.65)-(4.66),(4.69)-(4.70)$, and (4.73), and

$$
\sigma=\frac{s}{p_{0} t_{0}} \exp \left(-\frac{a \tau}{\rho_{0} x_{0}}\right) .
$$

Again, it is seen that the solution (4.74)-(4.75), (4.65)-(4.66), (4.69)-(4.70), and (4.73) is also the solution of $\operatorname{ODE}(5.6)-(5.7)$ of Sachdev and Reddy [1], namely,

$$
(W-a \sigma) \frac{d W}{d \sigma}-c_{*} P^{-1 /\left(\gamma^{\prime}-1\right)} \frac{d P}{d \sigma}=a\left(1-W^{\prime}\right),
$$




$$
P \frac{d W}{d \sigma}-(W-a \sigma) \frac{d P}{d \sigma}=0, \quad P=H^{\gamma /(\gamma-1)} .
$$

We may note that the series (4.74)-(4.75) with (4.65)-(4.66), (4.69)-(4.70), and (4.73) cannot be summed up; the system of ODE (4.77)-(4.78) is also not soluble in a closed form.

The parametric representation of the shock may be found from (3.8), (4.64), and (2.11) to be

$$
\frac{x}{x_{0}}=\frac{1}{2 a}\left[\exp \left(\frac{2 a \tau}{\rho_{0} x_{0}}\right)-1\right], \quad \frac{t}{t_{0}}=\frac{1}{a}\left[\exp \left(\frac{a \tau}{\rho_{0} x_{0}}\right)-1\right] .
$$

The trajectory of the shock wave, shock velocity, and the undisturbed density are therefore given, respectively, by

$$
\begin{gathered}
\frac{x(t)}{x_{0}}=\frac{1}{2 a}\left[\left(1+\frac{a t}{t_{0}}\right)^{2}-1\right], \\
U(t)=\frac{x_{0}}{t_{0}}\left(1+\frac{a t}{t_{0}}\right), \\
\rho_{*}(x)=\rho_{0}\left(1+\frac{2 a x}{x_{0}}\right)^{-1} .
\end{gathered}
$$

The functions $x$ and $t$ are found to be

$$
\begin{aligned}
\frac{x(H, \tau)}{x_{0}}= & \frac{\exp \left(2 a \tau / \rho_{0} x_{0}\right)}{a b_{0}} \int_{H_{*}}^{H}\left[\frac{\gamma-1}{\gamma} H\left(\frac{d W}{d H}\right)^{2}-\frac{\gamma c_{*}}{\gamma-1}\right] H^{-\gamma /(\gamma-1)} d H \\
& +\frac{1}{2 a}\left[\exp \left(\frac{2 a \tau}{\rho_{0} x_{0}}\right)-1\right], \\
\frac{t(H, \tau)}{t_{0}}= & \frac{\exp \left(a \tau / \rho_{0} x_{0}\right)}{a} \int_{H_{*}}^{H}\left[\frac{\gamma-1}{\gamma} H\left(\frac{d W}{d H}\right)^{2}-\frac{\gamma c_{*}}{\gamma-1}\right] \frac{H^{-\gamma /(\gamma-1)}}{(W-1)} d H \\
& +\frac{1}{a}\left[\exp \left(\frac{a \tau}{\rho_{0} x_{0}}\right)-1\right],
\end{aligned}
$$

where $H_{*} \leq H<\infty$ for $a=1$, and $1<H \leq H_{*}$ for $a=-1$. The piston path, which corresponds to $\tau=0$, is given by

$$
\begin{aligned}
\frac{X(H)}{x_{o}} & =\frac{1}{a b_{0}} \int_{H_{*}}^{H}\left[\frac{\gamma-1}{\gamma} H\left(\frac{d W}{d H}\right)^{2}-\frac{\gamma c_{*}}{\gamma-1}\right] H^{-\gamma /(\gamma-1)} d H, \\
\frac{t(H)}{t_{0}} & =\frac{1}{a} \int_{H_{*}}^{H}\left[\frac{\gamma-1}{\gamma} H\left(\frac{d W}{d H}\right)^{2}-\frac{\gamma c_{*}}{\gamma-1}\right] \frac{H^{-\gamma /(\gamma-1)}}{(W-1)} d H .
\end{aligned}
$$

The solution (4.74)-(4.75) with (4.65)-(4.66), (4.69)-(4.70) and (4.73) of PDE (4.1)(4.2) thus involves four arbitrary constants: $x_{0}, t_{0}, p_{0}$, and $\rho_{0}$. The interpretation of the above solution is as follows. A piston moves according to (4.85) and gives rise to a shock whose trajectory is given by (4.80). For $a>0 \quad(<0)$ the undisturbed density vanishes and the shock velocity grows without bound in an infinite distance and infinite time, respectively $\left(\rho_{*}\right.$ becomes infinite in a finite distance $x=x_{0} / 2$ while the shock velocity decays to zero in time $t=t_{0}$ ). 
5. Solutions of the system (3.20)-(3.21) with shocks of finite strength. With

$$
W=\frac{\rho_{*} u^{2}}{2}, \quad H=\left(\frac{p}{p_{0}}\right)^{(\gamma-1) / \gamma}
$$

as new independent variables, Eqs. (3.20)-(3.21) may be rewritten as

$$
\begin{gathered}
\rho_{*} H_{\tau}+\left(W+\frac{\beta f \rho_{*}}{\gamma-1} H+\rho_{*} \phi\right) H_{s}-\frac{\gamma-1}{\gamma} H W_{s}=0, \\
\rho_{*} W_{\tau}+\left(W+\frac{\beta f \rho_{*}}{\gamma-1} H+\rho_{*} \phi\right) W_{s}-\frac{2 \beta f \rho_{*}}{\gamma-1} W H_{s}-W \frac{d \rho_{*}}{d \tau}=0,
\end{gathered}
$$

where $\beta=p_{0}^{(\gamma-1) / \gamma}$. The shock conditions (2.8)-(2.10) and (3.12) now become

$$
\begin{gathered}
W_{s=0}=\frac{\left(P_{*}-1\right)^{2} p_{0}}{\left((\gamma+1) P_{*}+\gamma-1\right)}, \\
H_{s=0}=P_{*}^{(\gamma-1) / \gamma}, \\
f=\frac{\left(p_{0} P_{*}\right)^{1 / \gamma}\left[(\gamma-1) P_{*}+\gamma+1\right]}{\rho_{*}\left[(\gamma+1) P_{*}+\gamma-1\right]}, \\
U=\left\{\frac{p_{0}}{2 \rho_{*}}\left[(\gamma+1) P_{*}+\gamma-1\right]\right\}^{1 / 2},
\end{gathered}
$$

where $P_{*}=p / p_{0}$. Equations (5.4)-(5.7) suggest that if we choose the functions $f, \rho_{*}$, and $U$ at the shock such that $f \rho_{*}=$ constant and $\rho_{*} U^{2}=$ constant, the variables $W$ and $H$ just behind the shock (or $P_{*}$ ) become constant. Thus, we have

$$
\frac{f \rho_{*} \beta}{\gamma-1}=\frac{p_{0} P_{*}^{1 / \gamma}\left[(\gamma-1) P_{*}+\gamma+1\right]}{(\gamma-1)\left[(\gamma+1) P_{*}+\gamma-1\right]}=a_{0}
$$

say, and

$$
\phi \rho_{*}=-\frac{p_{0}}{\gamma-1}
$$

(see (3.17)). Equations (5.2)-(5.3) now become

$$
\begin{gathered}
\rho_{*} H_{\tau}+\left(W+a_{0} H+a_{1}\right) H_{s}-\frac{\gamma-1}{\gamma} H W_{s}=0, \\
\rho_{*} W_{\tau}+\left(W+a_{0} H+a_{1}\right) W_{s}-2 a_{0} W H_{s}-W \frac{d \rho_{*}}{d \tau}=0 .
\end{gathered}
$$

We look for a solution of the system (5.10)-(5.11) in series form

$$
\begin{aligned}
& W(\tau, s)=\sum_{i=0}^{\infty} W^{(j)}(\tau) \frac{s^{j}}{j !}, \\
& H(\tau, s)=\sum_{i=0}^{\infty} H^{(j)}(\tau) \frac{s^{j}}{j !} .
\end{aligned}
$$


Substituting (5.12)-(5.13) into Eqs. (5.10)-(5.11) and putting the coefficients of $s^{i}$, $i \geq 0$, equal to zero, we get

$$
\begin{array}{r}
W^{(n+1)}(\tau)=\frac{\gamma}{(\gamma-1) H^{(0)}}\left[\left(W^{(0)}+a_{0} H^{(0)}+a_{1}\right) H^{(n+1)}+\rho_{*} \frac{d H^{(n)}}{d \tau}\right. \\
\left.+\tau^{(n)}\left(\left\{W^{(i)}\right\},\left\{H^{(i)}\right\}\right)\right], \\
H^{(n+1)}(\tau)=\frac{1}{D_{0}}\left[\rho_{*} \frac{d W^{(n)}}{d \tau}-W^{(n)} \frac{d \rho_{*}}{d \tau}+\frac{\gamma \rho_{*}}{(\gamma-1) H^{(0)}}\left(W^{(0)}\right.\right. \\
\left.\left.+a_{0} H^{(0)}+a_{1}\right) \frac{d H^{(n)}}{d \tau}+S^{(n)}\left(\left\{W^{(i)}\right\},\left\{H^{(i)}\right\}\right)\right], \\
n=0,1, \ldots,
\end{array}
$$

where

$$
\begin{aligned}
T^{(n)}\left(\left\{W^{(i)}\right\},\left\{H^{(i)}\right\}\right)= & \sum_{k=1}^{n}\left(\begin{array}{l}
n \\
k
\end{array}\right)\left[\left(W^{(k)}+a_{0} H^{(k)}\right) H^{(n+1-k)}-\frac{\gamma-1}{\gamma} H^{(k)} W^{(n+1-k)}\right], \\
S^{(n)}\left(\left\{W^{(i)}\right\},\left\{H^{(i)}\right\}\right)= & \sum_{k=1}^{n}\left(\begin{array}{l}
n \\
k
\end{array}\right)\left[\left(W^{(k)}+a_{0} H^{(k)}\right) W^{(n+1-k)}-2 a_{0} W^{(k)} H^{(n+1-k)}\right] \\
& +\frac{\gamma\left(W^{(0)}+a_{0} H^{(0)}+a_{1}\right)}{(\gamma-1) H^{(0)}} T^{(n)}\left(\left\{W^{(i)}\right\},\left\{H^{(i)}\right\}\right), \\
D_{0}= & 2 a_{0} W^{(0)}-\frac{\gamma\left(W^{(0)}+a_{0} H^{(0)}+a_{1}\right)^{2}}{(\gamma-1) H^{(0)}} .
\end{aligned}
$$

As in Sec. 4, we take $W^{(0)}$ and $H^{(0)}$ as given, respectively, by (5.4) and (5.5), so that the series solution (5.12)-(5.13) satisfies the conditions at the shock; the first and all higher-order coefficients may be obtained through their algebraic dependence on the lower-order coefficients (see (5.14)-(5.15)).

Now we choose $\rho_{*}(\tau)$ and $f_{*}(\tau)$ to derive the power-law type and exponential type similarity solutions from the series solution (5.12)-(5.13), (5.4)-(5.5), and (5.14)(5.16), as special cases.

(I) Power-law similarity solutions of the system (5.2)-(5.3). We assume

$$
\begin{gathered}
\rho_{*}=\rho_{0}\left(1+\frac{a \tau}{\rho_{0} x_{0}}\right)^{-\alpha}, \\
f=\frac{\gamma p_{0}^{1 / \gamma}}{b_{0} \rho_{0}} c_{*}\left(1+\frac{a \tau}{\rho_{0} x_{0}}\right)^{\alpha},
\end{gathered}
$$

where $p_{0}, \rho_{0}, x_{0}$, and $t_{0}$ are arbitrary constants, with dimensions of pressure, density, distance and time, respectively. $\alpha(\neq 0)$ is the exponent in the similarity variable. The constant $a$ is either +1 or -1 . 
The parameter $c_{*}$ is defined as

$$
c_{*}=\frac{b_{0}\left[\gamma-1+2 \gamma / b_{0}\right]}{\gamma(\gamma+1)} P_{*}^{1 / \gamma}
$$

where

$$
P_{*}=\frac{2 b_{0}-\gamma+1}{\gamma+1}
$$

and $b_{0}=\rho_{0} x_{0}^{2} /\left(p_{0} t_{0}^{2}\right)$. Now we note from (5.8) that

$$
a_{0}=\frac{p_{0} P_{*}^{1 / \gamma}\left[\gamma-1+2 \gamma / b_{0}\right]}{\gamma^{2}-1}
$$

In consequence of the above, we have

$$
\begin{gathered}
H^{(0)}=H_{s=0}=P_{*}^{(\gamma-1) / \gamma}, \\
W^{(0)}=W_{s=0}=\frac{2 p_{0}}{b_{0}}\left(\frac{b_{0}-\gamma}{\gamma+1}\right)^{2}
\end{gathered}
$$

from (5.4)-(5.5). Equations (5.15)-(5.16) then yield

$$
\begin{aligned}
W^{(n+1)}(z) & =W_{*}^{(n+1)} z^{n+1}, \\
H^{(n+1)}(z) & =H_{*}^{(n+1)} z^{n+1}, \quad n=0,1,2, \ldots,
\end{aligned}
$$

where

$$
z=\frac{1}{x_{0}}\left(1+\frac{a \tau}{\rho_{0} x_{0}}\right)^{-(\alpha+1)}
$$

and the constants $W_{*}^{(n+1)}$ and $H_{*}^{(n+1)}, n \geq 0$, are given by

$$
\begin{array}{r}
W_{*}^{(n+1)}=\frac{\gamma}{(\gamma-1) H^{(0)}}\left[\left(W^{(0)}+a_{0} H^{(0)}+a_{1}\right) H_{*}^{(n+1)}-a n(\alpha+1) H_{*}^{(n)}\right. \\
\left.+T_{*}^{(n)}\left(\left\{W_{*}^{(i)}\right\},\left\{H_{*}^{(i)}\right\}\right)\right], \\
H_{*}^{(n+1)}=\frac{1}{D_{*}}\left[a(\alpha-n(\alpha+1)) W_{*}^{(n)}-\frac{a n \gamma(\alpha+1)}{(\gamma-1) H^{(0)}}\left(W^{(0)}+a_{0} H^{(0)}+a_{1}\right) H_{*}^{(n)}\right. \\
\left.+S_{*}^{(n)}\left(\left\{W_{*}^{(i)}\right\},\left\{H_{*}^{(i)}\right\}\right)\right],
\end{array}
$$


with

$$
\begin{aligned}
T_{*}^{(n)}\left(\left\{W_{*}^{(i)}\right\},\left\{H_{*}^{(i)}\right\}\right)= & \sum_{k=1}^{n}\left(\begin{array}{l}
n \\
k
\end{array}\right)\left[\left(W_{*}^{(k)}+a_{0} H_{*}^{(k)}\right) H_{*}^{(n+1-k)}-\frac{\gamma-1}{\gamma} H_{*}^{(k)} W_{*}^{(n+1-k)}\right], \\
S_{*}^{(n)}\left(\left\{W_{*}^{(i)}\right\},\left\{H_{*}^{(i)}\right\}\right)= & \sum_{k=1}^{n}\left(\begin{array}{l}
n \\
k
\end{array}\right)\left[\left(W_{*}^{(k)}+a_{0} H_{*}^{(k)}\right) W_{*}^{(n+1-k)}-2 a_{0} W_{*}^{(k)} H_{*}^{(n+1-k)}\right] \\
& +\frac{\gamma\left(W^{(0)}+a_{0} H^{(0)}+a_{1}\right)}{(\gamma-1) H^{(0)}} T_{*}^{(n)}\left(\left\{W_{*}^{(i)}\right\},\left\{H_{*}^{(i)}\right\}\right), \\
D_{*}= & 2 a W^{(0)}-\frac{\gamma\left(W^{(0)}+a_{0} H^{(0)}+a_{1}\right)^{2}}{(\gamma-1) H^{(0)}} .
\end{aligned}
$$

The form (5.24)-(5.25) for the first and higher order coefficients of the series solution (5.12)-(5.13) may be proved by an induction argument. The series (5.12)-(5.13) now becomes

$$
\begin{aligned}
& W=\sum_{j=0}^{\infty} W_{*}^{(j)} \frac{\sigma^{j}}{j !}, \\
& H=\sum_{j=0}^{\infty} H_{*}^{(j)} \frac{\sigma^{j}}{j !}
\end{aligned}
$$

where

$$
\sigma=\frac{s}{x_{0}}\left(1+\frac{a \tau}{\rho_{0} x_{0}}\right)^{-(\alpha+1)},
$$

and $W_{*}^{(0)}=W^{(0)}$ and $H_{*}^{(0)}=H^{(0)}$. The coefficients in the series $(5.28)-(5.29)$ are given by (5.22)-(5.23) and (5.26)-(5.27) with $j=n+1, n=0,1, \ldots$ We note that (5.28)-(5.29), (5.22)-(5.23), and (5.26)-(5.27) is also a solution of the ODE

$$
\begin{array}{r}
\left(-a(\alpha+1) \sigma+W+a_{0} H+a_{1}\right) \frac{d H}{d \sigma}-\frac{\gamma-1}{\gamma} H \frac{d W}{d \sigma}=0, \\
\left(-a(\alpha+1) \sigma+W+a_{o} H+a_{1}\right) \frac{d W}{d \sigma}-2 a_{0} W \frac{d H}{d \sigma}+a \alpha W=0,
\end{array}
$$

with initial conditions $(5.22)-(5.23)$ at the shock. Here, $\sigma$ is the similarity variable defined by (5.30).

For a shock moving into a quiescent gas, Eq. (3.18) gives

$$
d \tau=\rho_{*} d x
$$

which, on using (5.17) and integrating, yields

$$
\frac{x}{x_{0}}= \begin{cases}\frac{1}{a(\alpha+1)}\left[\left(1+\frac{a \tau}{\rho_{0} x_{0}}\right)^{\alpha+1}-1\right], & \alpha \neq-1, \\ \frac{1}{a} \ln \left[1+\frac{a \tau}{\rho_{0} x_{0}}\right], & \alpha=-1 .\end{cases}
$$

Using (5.7), (5.33), and (5.17) in Eq. (2.11) and integrating, we have

$$
\frac{t}{t_{0}}= \begin{cases}\frac{2}{a(\alpha+2)}\left[\left(1+\frac{a \tau}{\rho_{0} x_{0}}\right)^{(\alpha+2) / 2}-1\right], & \alpha \neq-2, \\ \frac{1}{a} \ln \left[1+\frac{a \tau}{\rho_{0} x_{0}}\right], & \alpha=-2 .\end{cases}
$$


The boundary conditions used to obtain (5.34) and (5.35) are $\tau=0$ when $x=$ $0, t=0$. Thus, the shock can be parametrically represented by Eqs. (5.34)-(5.35). The shock locus in the $(x, t)$-plane is given by

$$
\frac{x(t)}{x_{0}}= \begin{cases}\frac{1}{a(1+\alpha)}\left[\left(1+\frac{a(\alpha+2) t}{2 t_{0}}\right)^{2(\alpha+1) /(\alpha+2)}-1\right], & \alpha \neq-1,-2, \\ \frac{2}{a} \ln \left[1+\frac{a t}{2 t_{0}}\right], & \alpha=-1, \\ \frac{1}{a}\left(1-\exp \left(-\frac{a t}{t_{0}}\right)\right), & \alpha=-2 .\end{cases}
$$

The undisturbed density and the shock velocity are found to be

and

$$
\rho_{*}(x)= \begin{cases}\rho_{0}\left[1+\frac{a(\alpha+1) x}{x_{0}}\right]^{-\alpha /(\alpha+1)}, & \alpha \neq-1, \\ \rho_{0} \exp \left(\frac{a x}{x_{0}}\right), & \alpha=-1,\end{cases}
$$

We also have $b_{0}>\gamma$ in the present case.

$$
U(t)= \begin{cases}\frac{x_{0}}{t_{0}}\left[1+\frac{a(\alpha+2) t}{x_{0}}\right]^{-\alpha /(\alpha+2)}, & \alpha \neq-2, \\ \frac{x_{0}}{t_{0}} \exp \left(-\frac{a t}{t_{0}}\right), & \alpha=-2 .\end{cases}
$$

The functions $x$ and $t$ may be found to be

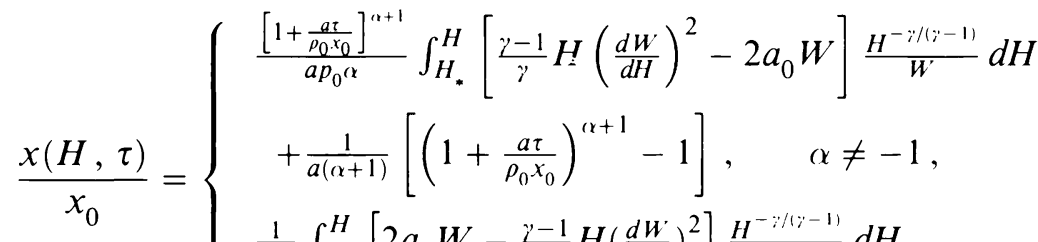

$$
\begin{aligned}
& \frac{1}{a p_{0}} \int_{H .}^{H}\left[2 a_{0} W-\frac{\eta-1}{\gamma} H\left(\frac{d W}{d H}\right)^{2}\right] \frac{H^{-\xi /(\gamma-1)}}{W} d H \\
& +\frac{1}{a} \ln \left[1+\frac{a \tau}{\rho_{0} x_{0}}\right], \quad \alpha=-1,
\end{aligned}
$$

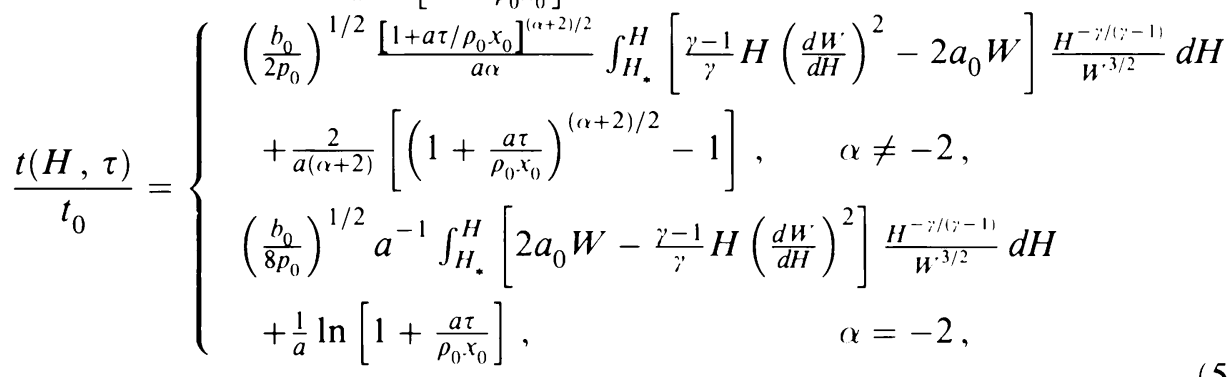

where $H_{*} \leq H<\infty$ if $a_{\alpha}>0$, and $1<H \leq H_{*}$ if $a_{\alpha}<0$.

Equations (5.39)-(5.40) with $\tau=$ constant give the parametric representation of the particle lines. The law of motion of the piston obtained by setting $\tau=0$ in (5.39) $-(5.40)$ is

$$
\begin{aligned}
& \frac{x(H)}{x_{0}}=\frac{1}{a p_{0} \alpha} \int_{H_{.}}^{H}\left[\frac{\gamma-1}{\gamma} H\left(\frac{d W}{d H}\right)^{2}-2 a_{0} W\right] \frac{H^{-\gamma /(\gamma-1)}}{W} d H, \\
& \frac{t(H)}{t_{0}}=\left(\frac{b_{0}}{2 p_{0}}\right)^{1 / 2} \frac{1}{a_{\alpha}} \int_{H_{.}}^{H}\left[\frac{\gamma-1}{\gamma} H\left(\frac{d W}{d H}\right)^{2}-2 a_{0} W\right] \frac{H^{-\gamma /(\gamma-1)}}{W^{3 / 2}} d H .
\end{aligned}
$$


We may again note that this solution, for the problem posed in Sec. 4, involves five arbitrary constants: $x_{0}, t_{0}, \rho_{0}, p_{0}$, and $\alpha$.

The behavior of this solution according to (5.37)-(5.38) is as follows. When $0>$ $\alpha>-1$ and $a>0 \quad(<0)$, the undisturbed density, $\rho_{*}$, grows to infinity in an infinite distance while the shock velocity, $U$ decays to zero in an infinite time $\left(\rho_{*}\right.$ vanishes in finite distance $x=\left|x_{0} / a(\alpha+1)\right|$ and $U$ becomes infinite in a finite time $\left.t=\left|2 t_{0} / a(\alpha+2)\right|\right)$. When $-1>\alpha>-2$ and $a>0 \quad(<0), \rho_{*}$ increases and becomes infinite at a finite distance $x=\left|x_{0} / a(\alpha+1)\right|$ but the shock velocity vanishes in an infinite time ( $\rho_{*}$ decays to zero in an infinite distance and the shock velocity becomes infinite in finite time $\left.t=\left|2 t_{0} / a(\alpha+2)\right|\right)$. For $-2>\alpha>-\infty$ and $a>0 \quad(<0), \rho_{*}$ becomes infinite at finite distance $x=\left|x_{0} / a(\alpha+1)\right|$ and $U$ vanishes in finite time $t=\left|2 t_{0} / a(\alpha+2)\right| \quad\left(\rho_{*}\right.$ decays to zero and $U$ increases without bound in an infinite distance and infinite time, respectively). For $\alpha=-1$ and $a>0$ $(<0), \rho_{*}$ grows exponentially and $U$ vanishes in an infinite distance and infinite time, respectively $\left(\rho_{*}\right.$ decays exponentially to zero in an infinite distance, while $U$ becomes infinite in finite time $\left.t=2 t_{0}\right)$. For $\alpha=-2$ and $a>0 \quad(<0), \rho_{*}$ grows to infinity in a finite distance $x=x_{0}$, and $U$ becomes zero exponentially in an infinite time $\left(\rho_{*}\right.$ becomes infinite in an infinite distance and $U$ grows exponentially to infinity in an infinite time).

We remark that the solution of the system of ODE (5.31)-(5.32) may be found in a form involving a quadrature, provided $\alpha=-1$. In this case, if we assume that $W$ is a function of $H$, that is,

$$
W=Q(H)
$$

then Eqs. (5.22)-(5.23) give

$$
W^{(0)}=Q\left(H^{(0)}\right)=\frac{2 p_{0}}{b_{0}}\left(\frac{b_{0}-\gamma}{\gamma+2}\right)^{2},
$$

while Eqs. (5.26) $-(5.27)$ yield

$$
\begin{aligned}
& \left(\frac{d Q}{d H}\right)_{H=H^{(0)}}=\left(W^{(0)}+a_{0} \gamma H^{(0)}+a_{1}\right) \frac{\gamma\left(H^{(0)}\right)^{-1}}{\gamma-1}, \\
& \left(\frac{d^{2} Q}{d H^{2}}\right)_{H=H^{(0)}}=\frac{\gamma\left(W^{(0)}+a_{0} \gamma H^{(0)}+a_{1}\right)}{(\gamma-1)^{2}}\left(H^{(0)}\right)^{-2}, \\
& \left(\frac{d^{3} Q}{d H^{3}}\right)_{H=H^{(0)}}=\frac{\gamma(2-\gamma)}{(\gamma-1)^{3}}\left(W^{(0)}+a_{0} H^{(0)}+a_{1}\right)\left(H^{(0)}\right)^{-3},
\end{aligned}
$$

for $n=0,1,2, \ldots$. In view of $(5.43)-(5.46)$ and $(5.28)-(5.29)$, we find that

$W=Q(H)=-a_{1}-\gamma a_{0} H+\left(W^{(0)}+a_{0} \gamma H^{(0)}+a_{1}\right)\left(\frac{H}{H^{(0)}}\right)^{\gamma /(\gamma-1)}+(\gamma-1) a_{0} \frac{H^{\gamma /(\gamma-1)}}{\left(H^{(0)}\right)^{1 /(\gamma-1)}}$. 


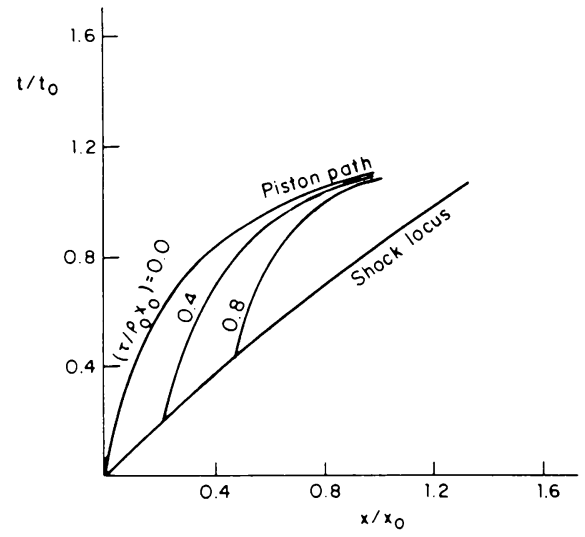

Fig. 1. Piston path, particle trajectories, and shock locus with $\alpha=0.5, a=1, b_{0}=1.8, \gamma=1.5$

Thus, for $\alpha=-1$, the solution may be written as

$$
\begin{gathered}
u=\left[\frac{2}{\rho_{*}} W\right]^{1 / 2}, \quad \rho_{*}=\rho_{0} \exp \left(\frac{a x}{x_{0}}\right), \\
f=\frac{\gamma p_{0}^{1 / \gamma}}{b_{0} \rho_{0}} c_{*}\left(1+\frac{a \tau}{\rho_{0} x_{0}}\right)^{-1}, \quad U=\frac{x_{0}}{t_{0}}\left(1+\frac{a t}{2 t_{0}}\right)^{-1}, \\
\frac{x(t)}{x_{0}}=\frac{1}{a} \ln \left[1+\frac{a t}{2 t_{0}}\right], \\
\frac{x}{x_{0}}=\frac{1}{a p_{0}} \int_{H_{*}}^{H}\left[2 a_{0} W-\frac{\gamma-1}{\gamma} H\left(\frac{d W}{d H}\right)^{2}\right] \frac{H^{-\gamma(\gamma-1)}}{W} d H, \\
\frac{t}{t_{0}}=\left(\frac{b_{0}}{2 p_{0}}\right)^{1 / 2} a^{-1} \int_{H_{*}}^{H}\left[2 a_{0} W-\frac{\gamma-1}{\gamma} H\left(\frac{d W}{d H}\right)^{2}\right] \frac{H^{-\gamma /(\gamma-1)}}{W^{3 / 2}} d H,
\end{gathered}
$$

where $W$ is given by (5.47), and $H_{*} \leq H<\infty$ if $a=-1$, and $1<H \leq H_{*}$ if $a=1$. Fig. 1 shows the piston motion, particle trajectories, and shock locus for $\gamma=1.5, b_{0}=1.8, \alpha=-0.5, a=-1$. For these constants, the undisturbed density becomes zero at $x=2 x_{0}$, while the shock velocity becomes infinite at time $t=4 t_{0} / 3$.

(II) Exponential type similarity solutions for the system (5.2)-(5.3). For now we choose

$$
\begin{gathered}
\rho_{*}=\rho_{0} \exp \left(-\frac{a \tau}{\rho_{0} x_{0}}\right), \\
f=\frac{\gamma p_{0}^{1 / \gamma}}{b_{0} \rho_{0}} c_{*} \exp \left(\frac{a \tau}{\rho_{0} x_{0}}\right),
\end{gathered}
$$

where the constants $p_{0}, \rho_{0}, x_{0}, t_{0}, a, b_{0}$, and $c_{*}$ are the same as for power-law type similarity solutions (see Eqs. (5.19)-(5.20)). We also choose $a_{0}$ to be given by (5.21), and the zeroth-order solution in (5.12)-(5.13) by (5.22)-(5.23). 
Using (5.49)-(5.50) in (5.14)-(5.16), we obtain

$$
\begin{aligned}
W^{(n+1)}(z) & =W_{*}^{(n+1)} z^{n+1}, \\
H^{(n+1)}(z) & =H_{*}^{(n+1)} z^{n+1}, \quad n=0,1,2, \ldots,
\end{aligned}
$$

where

$$
z=\frac{1}{x_{0}} \exp \left(-\frac{a \tau}{\rho_{0} x_{0}}\right)
$$

and

$$
\begin{aligned}
& W_{*}^{(n+1)}=\frac{\gamma}{(\gamma-1) H^{(0)}}\left[\left(W^{(0)}+a_{0} H^{(0)}+a_{1}\right) H_{*}^{(n+1)}-a n H_{*}^{(n)}+T_{*}^{(n)}\left(\left\{W_{*}^{(i)}\right\},\left\{H_{*}^{(i)}\right\}\right)\right], \\
& H_{*}^{(n+1)}=\frac{1}{D_{*}}\left[a(1-n) W_{*}^{(n)}-\frac{\gamma a n\left(W^{(0)}+a_{0} H^{(0)}+a_{1}\right)}{(\gamma-1) H^{(0)}} H_{*}^{(n)}+S_{*}^{(n)}\left(\left\{W_{*}^{(i)}\right\},\left\{H_{*}^{(i)}\right\}\right)\right]
\end{aligned}
$$

with

$$
\begin{aligned}
T_{*}^{(n)}\left(\left\{W_{*}^{(i)}\right\},\left\{H_{*}^{(i)}\right\}\right)= & \sum_{k=1}^{n}\left(\begin{array}{l}
n \\
k
\end{array}\right)\left[\left(W_{*}^{(k)}+a_{0} H_{*}^{(k)}\right) H_{*}^{(n+1-k)}-\frac{\gamma-1}{\gamma} H_{*}^{(k)} W_{*}^{(n+1-k)}\right], \\
S_{*}^{(n)}\left(\left\{W_{*}^{(i)}\right\},\left\{H_{*}^{(i)}\right\}\right)= & \sum_{k=1}^{n}\left(\begin{array}{l}
n \\
k
\end{array}\right)\left[\left(W_{*}^{(k)}+a_{0} H_{*}^{(k)}\right) W_{*}^{(n-1+k)}-2 a_{0} W_{*}^{(k)} H_{*}^{(n+1-k)}\right] \\
& +\frac{\gamma\left(W^{(0)}+a_{0} H^{(0)}+a_{1}\right)}{(\gamma-1) H^{(0)}} T_{*}^{(n)}\left(\left\{W_{*}^{(i)}\right\},\left\{H_{*}^{(i)}\right\}\right), \\
D_{*}= & 2 a_{0} W^{(0)}-\frac{\gamma\left(W^{(0)}+a_{0} H^{(0)}+a_{1}\right)^{2}}{(\gamma-1) H^{(0)}} .
\end{aligned}
$$

Now the series solution (5.12)-(5.13) may be rewritten as

$$
\begin{gathered}
W=\sum_{j=0}^{\infty} W_{*}^{(j)} \frac{\sigma^{j}}{j !}, \\
H=\sum_{j=0}^{\infty} H_{*}^{(j)} \frac{\sigma^{j}}{j !},
\end{gathered}
$$

where

$$
\sigma=\frac{s}{x_{0}} \exp \left(-\frac{a \tau}{\rho_{0} x_{0}}\right)
$$

and $W_{*}^{(j)}$ and $H_{*}^{(j)}, j \geq 0$, are given by $(5.22)-(5.23)$ and $(5.54)-(5.55)$.

We find that the solution (5.56)-(5.57), (5.22)-(5.23), and (5.54)-(5.55) is also the solution of the ODE, which results from (5.10)-(5.11) using the similarity ansatz (5.58), namely

$$
\begin{gathered}
{\left[W+a_{0} H+a_{1}-a \sigma\right] \frac{d H}{d \sigma}-\frac{\gamma-1}{\gamma} H \frac{d W}{d \sigma}=0,} \\
{\left[W+a_{0} H+a_{1}-a \sigma\right] \frac{d W}{d \sigma}-2 a_{0} W \frac{d H}{d \sigma}+a W=0 .}
\end{gathered}
$$


The parametric representation of the shock is

$$
\begin{aligned}
& \frac{x}{x_{0}}=\frac{1}{a}\left[\exp \left(\frac{a \tau}{\rho_{0} x_{0}}\right)-1\right], \\
& \frac{t}{t_{0}}=\frac{2}{a}\left[\exp \left(\frac{a \tau}{2 \rho_{0} x_{0}}\right)-1\right] .
\end{aligned}
$$

The shock trajectory, shock velocity, and the undisturbed density are, respectively, given by

$$
\begin{gathered}
\frac{x}{x_{0}}=\frac{1}{a}\left[\left(1+\frac{a t}{2 t_{0}}\right)^{2}-1\right], \\
U=\frac{x_{0}}{t_{0}}\left[1+\frac{a t}{2 t_{0}}\right], \\
\rho_{*}=\rho_{0}\left[1+\frac{a x}{x_{0}}\right]^{-1} .
\end{gathered}
$$

The functions $x(t, H)$ and $t(t, H)$ are given by

$$
\begin{aligned}
\frac{x(\tau, H)}{x_{0}}= & \frac{\exp \left(a \tau / \rho_{0} x_{0}\right)}{a p_{0}} \int_{H_{*}}^{H}\left[\frac{\gamma-1}{\gamma} H\left(\frac{d W}{d H}\right)^{2}-2 a_{0} W\right] \frac{H^{-\gamma /(\gamma-1)}}{W} d H \\
& +\frac{1}{a}\left[\exp \left(\frac{a \tau}{\rho_{0} x_{0}}\right)-1\right], \\
\frac{t(\tau, H)}{t_{0}}= & \left(\frac{b_{0}}{2 p_{0}}\right)^{1 / 2} \frac{\exp \left(a \tau / 2 \rho_{0} x_{0}\right)}{a} \int_{H_{*}}^{H}\left[\frac{\gamma-1}{\gamma} H\left(\frac{d W}{d H}\right)^{2}-2 a_{0} W\right] \frac{H^{-\gamma /(\gamma-1)}}{W^{3 / 2}} d H \\
+ & \frac{2}{a}\left[\exp \left(\frac{a \tau}{2 \rho_{0} x_{0}}\right)-1\right],
\end{aligned}
$$

where $H_{*} \leq H<\infty$ if $a=1$, and $1<H \leq H_{*}$ if $a=-1$.

Setting $\tau=0$ in (5.66)-(5.67) we obtain the parametric representation of the piston path:

$$
\begin{aligned}
\frac{x(H)}{x_{0}} & =\frac{1}{p_{0} a} \int_{H_{*}}^{H}\left[\frac{\gamma-1}{\gamma} H\left(\frac{d W}{d H}\right)^{2}-2 a_{0} W\right] \frac{H^{-\gamma /(\gamma-1)}}{W} d H, \\
\frac{t(H)}{t_{0}} & =\left(\frac{b_{0}}{2 p_{0}}\right)^{1 / 2} \frac{1}{a} \int_{H_{*}}^{H}\left[\frac{\gamma-1}{\gamma} H\left(\frac{d W}{d H}\right)^{2}-2 a_{0} W\right] \frac{H^{-\gamma /(\gamma-1)}}{W^{3 / 2}} d H .
\end{aligned}
$$

It may be noted that when $a=1 \quad(-1)$, the undisturbed density decreases (increases) with $x$, the shock velocity increases (decreases) with $t$, and the nondimensional pressure $P=H^{\gamma /(\gamma-1)} \geq H_{*}^{\gamma /(\gamma-1)}\left(1<P \leq H_{*}^{\gamma /(\gamma-1)}\right)$ at the back of the shock. This solution, thus, involves four arbitrary constants $p_{0}, \rho_{0}, x_{0}$, and $t_{0}$.

Fig. 2 shows the piston motion, particle trajectories, and shock locus for $a=1$, $b_{0}=2, \gamma=1.4$. Here, the undisturbed density vanishes in an infinite distance, and the shock velocity becomes infinite at $t=\infty$. 


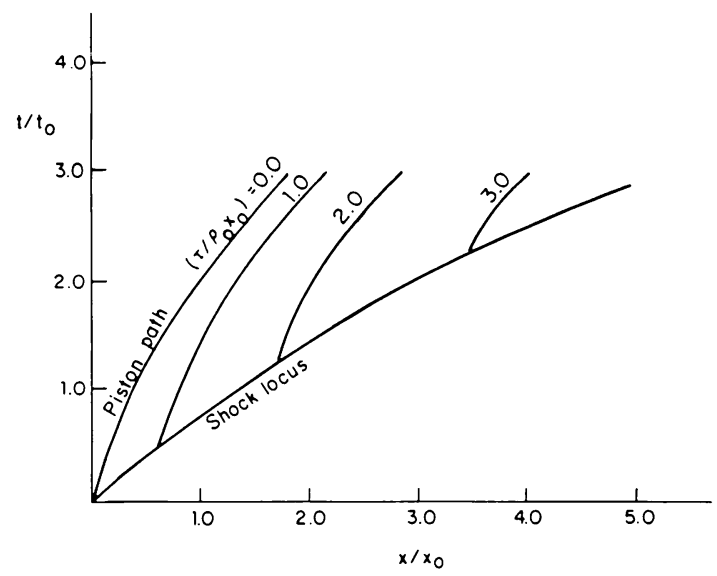

FIG. 2. Piston path, particle trajectories, and shock locus with $a=1, b_{0}=2, \gamma=1.4$

6. Solutions of $(3.23)-(3.24)$ with strong shocks. The introduction of the variables

$$
W=\frac{u}{\phi} \quad \text { and } \quad H=\left(\frac{p}{b_{0}}\right)^{(\gamma-1) / \gamma}
$$

in the basic system (3.23)-(3.24) leads to the system

$$
\begin{gathered}
H_{\tau}+\phi W H_{s}-\frac{\gamma-1}{\gamma} \phi H W_{s}=0, \\
\phi\left(W_{\tau}+\phi W W_{s}\right)-b_{0}^{(\gamma-1) / \gamma} f H_{s}+W \frac{d \phi}{d \tau}=0,
\end{gathered}
$$

where $\phi(\tau)$ is an arbitrary function of $\tau, b_{0}$ is an arbitrary constant with the dimension of pressure, and $f$ is given by (3.25).

Further, if we assume that

$$
\left.\phi=U, \quad \rho_{*} U^{2}=b_{0}=\frac{\rho_{0} x_{0}^{2}}{t_{0}^{2}} \quad \text { (a constant }\right),
$$

where $U$ is the shock velocity, the strong shock conditions (2.12)-(2.14) imply that

$$
W_{s=0}=\frac{2}{\gamma+1}
$$

and

$$
H_{s=0}=\left(\frac{2}{\gamma+1}\right)^{(\gamma-1) / \gamma}
$$

are constant.

We seek a solution of the system (6.2)-(6.3) in the series form

$$
\begin{aligned}
& W(s, \tau)=\sum_{j=0}^{\infty} W^{(j)}(\tau) \frac{s^{j}}{j !}, \\
& H(s, \tau)=\sum_{j=0}^{\infty} H^{(j)}(\tau) \frac{s^{j}}{j !} .
\end{aligned}
$$


Substituting (6.7)-(6.8) into (6.2)-(6.3) and equating the coefficients of $s^{j}, j \geq 0$, to zero, we get the following algebraic relations for first and higher order coefficients:

$$
\begin{array}{r}
W^{(n+1)}(\tau)=\frac{\gamma}{(\gamma-1) \phi H^{(0)}}\left[\phi W^{(0)} H^{(n+1)}+\frac{d H^{(n)}}{d \tau}+\phi T^{(n)}\left(\left\{W^{(i)}\right\},\left\{H^{(i)}\right\}\right)\right], \quad \\
H^{(n+1)}(\tau)=\frac{1}{D_{0}}\left[W^{(n)} \frac{d \phi}{d \tau}+\phi\left(\frac{d W^{(n)}}{d \tau}+\frac{\gamma}{\gamma-1} \frac{W^{(0)}}{H^{(0)}} \frac{d H^{(n)}}{d \tau}+\phi S^{(n)}\left(\left\{W^{(i)}\right\},\left\{H^{(j)}\right\}\right)\right)\right], \\
n=0,1, \ldots, \quad
\end{array}
$$

with

$$
\begin{aligned}
T^{(n)}\left(\left\{W^{(i)}\right\},\left\{H^{(i)}\right\}\right) & =\sum_{k=1}^{n}\left(\begin{array}{l}
n \\
k
\end{array}\right)\left[W^{(k)} H^{(n+1-k)}-\frac{\gamma-1}{\gamma} H^{(k)} W^{(n+1-k)}\right], \\
S^{(n)}\left(\left\{W^{(i)}\right\},\left\{H^{(i)}\right\}\right) & =\sum_{k=1}^{n}\left(\begin{array}{l}
n \\
k
\end{array}\right) W^{(k)} W^{(n+1-k)}+\frac{\gamma}{\gamma-1} \frac{W^{(0)}}{H^{(0)}} T^{(n)}\left(\left\{W^{(i)}\right\},\left\{H^{(i)}\right\}\right), \\
D_{0} & =b_{0}^{(\gamma-1) / \gamma} f-\frac{\gamma}{\gamma-1}\left(\frac{W^{(0)}}{H^{(0)}}\right)^{2} .
\end{aligned}
$$

The zeroth-order terms in the series solution (6.7)-(6.8) are given by (6.5)-(6.6). Therefore,

$$
\begin{gathered}
W^{(0)}=\frac{2}{\gamma+1}, \\
H^{(0)}=\left(\frac{2}{\gamma+1}\right)^{(\gamma-1) / \gamma} .
\end{gathered}
$$

In the solution (6.7)-(6.8) with (6.12)-(6.13) and (6.10)-(6.11) of PDE (6.2)-(6.3), the functions $\phi$ and $f$ will be suitably chosen to derive power-law type and exponential type similarity solutions.

(I) Power-law similarity solutions of the system (6.2)-(6.3). We assume that

$$
\begin{gathered}
\rho_{*}=\rho_{0}\left(1+\frac{a \tau}{\rho_{0} x_{0}}\right)^{-2 \alpha}, \\
\phi=\frac{x_{0}}{t_{0}}\left(1+\frac{a \tau}{\rho_{0} x_{0}}\right)^{\alpha}, \\
f=\frac{c_{*} x_{0}^{2}}{t_{0}^{2}}\left(1+\frac{a \tau}{\rho_{0} x_{0}}\right)^{2 \alpha},
\end{gathered}
$$

where $c_{*}$ is a parameter defined as

$$
c_{*}=\frac{1}{2}\left(\frac{2}{\gamma+1}\right)^{(\gamma+1) / \gamma} b_{0}^{-(\gamma+1) / \gamma} .
$$

Here, $\alpha(\neq 0)$ is the similarity exponent, and $x_{0}, t_{0}, \rho_{0}$ are arbitrary dimensional constants, representing distance, time, and density, respectively. The constant $a$ takes values +1 or -1 . 
The substitution of $(6.14)-(6.16)$ into $(6.9)-(6.11)$ yields

$$
\begin{aligned}
& W^{(n+1)}(z)=W_{*}^{(n+1)} z^{n+1}, \\
& H^{(n+1)}(z)=H_{*}^{(n+1)} z^{n+1}, \quad n=0,1, \ldots,
\end{aligned}
$$

where

$$
z=\frac{t_{0}}{\rho_{0} x_{0}^{2}}\left(1+\frac{a \tau}{\rho_{0} x_{0}}\right)^{-(\alpha+1)}
$$

and

$$
\begin{aligned}
& W_{*}^{(n+1)}=\frac{\gamma}{(\gamma-1) H^{(0)}}\left[W^{(0)} H_{*}^{(n+1)}-n a(\alpha+1) H_{*}^{(n)}+T_{*}^{(n)}\left(\left\{W_{*}^{(i)}\right\},\left\{H_{*}^{(i)}\right\}\right)\right], \\
& H_{*}^{(n+1)}=\frac{1}{D_{*}}\left[(\alpha-n(\alpha+1)) a W_{*}^{(n)}-\frac{\gamma a n(\alpha+1) W^{(0)}}{(\gamma-1) H^{(0)}} H_{*}^{(n)}+S_{*}^{(n)}\left(\left\{W_{*}^{(i)}\right\},\left\{H_{*}^{(i)}\right\}\right)\right]
\end{aligned}
$$

are constants, with

$$
\begin{aligned}
T_{*}^{(n)}\left(\left\{W_{*}^{(i)}\right\},\left\{H_{*}^{(i)}\right\}\right) & =\sum_{k=1}^{n}\left(\begin{array}{l}
n \\
k
\end{array}\right)\left[W_{*}^{(k)} H_{*}^{(n+1-k)}-\frac{\gamma-1}{\gamma} H_{*}^{(k)} W_{*}^{(n+1-k)}\right], \\
S_{*}^{(n)}\left(\left\{W_{*}^{(i)}\right\},\left\{H_{*}^{(i)}\right\}\right) & =\sum_{k=1}^{n}\left(\begin{array}{l}
n \\
k
\end{array}\right) W_{*}^{(k)} W_{*}^{(n+1-k)}+\frac{\gamma W^{(0)}}{(\gamma-1) H^{(0)}} T_{*}^{(n)}\left(\left\{W_{*}^{(i)}\right\},\left\{H_{*}^{(i)}\right\}\right), \\
D_{*} & =b_{0}^{(\gamma-1) / \gamma} c_{*}-\frac{\gamma}{\gamma-1} \frac{W_{*}^{(0)} 2}{H^{(0)}}=-\frac{1}{\gamma-1}\left(\frac{2}{\gamma+1}\right)^{1 / \gamma} .
\end{aligned}
$$

Now we may rewrite the solution series $(6.7)-(6.8)$ as

$$
\begin{gathered}
W=\sum_{j=0}^{\infty} W_{*}^{(j)} \frac{\sigma^{j}}{i !}, \\
H=\sum_{j=0}^{\infty} H_{*}^{(j)} \frac{\sigma^{j}}{j !},
\end{gathered}
$$

where

$$
\sigma=\frac{t_{0}}{\rho_{0} x_{0}^{2}}\left(1+\frac{a \tau}{\rho_{0} x_{0}}\right)^{-(x+1)} s .
$$

The coefficients $W_{*}^{(j)}$ and $H_{*}^{(j)}$ in the series (6.23)-(6.24), for $j \geq 0$, are given by (6.12)-(6.13) and (6.21)-(6.22) with $j=n+1, n=0,1, \ldots$. It may easily be checked that the solution (6.23)-(6.24) is also the solution of the ODE

$$
\begin{gathered}
{[-a(\alpha+1) \sigma+W] \frac{d H}{d \sigma}-\frac{\gamma-1}{\gamma} H \frac{d W}{d \sigma}=0,} \\
{[-a(\alpha+1) \sigma+W] \frac{d W}{d \sigma}-c_{*} b_{0}^{(\gamma-1) / \gamma} \frac{d H}{d \sigma}+a \alpha W=0}
\end{gathered}
$$


with initial conditions $(6.5)-(6.6)$ at the shock. The parametric representation of the shock may be found to be

$$
\begin{gathered}
\frac{x}{x_{0}}= \begin{cases}\frac{1}{a(1+2 \alpha)}\left[\left(1+\frac{a \tau}{\rho_{0} x_{0}}\right)^{2 \alpha+1}-1\right], & \alpha \neq-\frac{1}{2}, \\
\frac{1}{a} \ln \left[\frac{a \tau}{\rho_{0} x_{0}}\right], & \alpha=-\frac{1}{2} ;\end{cases} \\
\frac{t}{t_{0}}= \begin{cases}\frac{1}{a(\alpha+1)}\left[\left(1+\frac{a \tau}{\rho_{0} x_{0}}\right)^{\alpha+1}-1\right], & \alpha \neq-1, \\
\frac{1}{a} \ln \left[1+\frac{a \tau}{\rho_{0} x_{0}}\right], & \alpha=-1 .\end{cases}
\end{gathered}
$$

Eliminating $\tau$ between (6.28) and (6.29), we get the shock trajectory as

$$
\frac{x(t)}{x_{0}}= \begin{cases}\frac{1}{a(2 \alpha+1)}\left[\left(1+\frac{a(\alpha+1) t}{t_{0}}\right)^{(2 \alpha+1) /(\alpha+1)}-1\right], & \alpha \neq-1,-\frac{1}{2}, \\ \frac{1}{a}\left[1-\exp \left(-\frac{a t}{t_{0}}\right)\right], & \alpha=-1, \\ \frac{2}{a} \ln \left[1+\frac{a t}{2 t_{0}}\right], & \alpha=-\frac{1}{2} .\end{cases}
$$

The shock velocity and undisturbed density distribution are given, respectively, by

$$
\begin{gathered}
U= \begin{cases}\frac{x_{0}}{t_{0}}\left(1+\frac{a(\alpha+1) t}{t_{0}}\right)^{\alpha /(\alpha+1)}, & \alpha \neq-1, \\
\frac{x_{0}}{t_{0}} \exp \left(-\frac{a t}{t_{0}}\right), & \alpha=-1 ;\end{cases} \\
\rho_{*}= \begin{cases}\rho_{0}\left[1+\frac{a(2 \alpha+1) x}{x_{0}}\right]^{-2 \alpha /(2 \alpha+1)}, & \alpha \neq-\frac{1}{2}, \\
\rho_{0} \exp \left(\frac{a x}{x_{0}}\right), & \alpha=-\frac{1}{2} .\end{cases}
\end{gathered}
$$

The functions $x(H, \tau)$ and $t(H, \tau)$ may be found to have the form

$$
\begin{aligned}
& \frac{x(H, \tau)}{x_{0}}= \begin{cases}\frac{\left(1+a \tau / \rho_{0} x_{0}\right)^{2 \alpha+1}}{a \alpha} \int_{H_{*}}^{H}\left[\frac{\gamma-1}{\gamma} H\left(\frac{d W}{d H}\right)^{2}-c\right] H^{-\gamma /(\gamma-1)} d H \\
+\frac{1}{a(2 \alpha+1)}\left[\left(1+\frac{a \tau}{\rho_{0} x_{0}}\right)^{2(x+1}-1\right], \quad \alpha \neq-\frac{1}{2}, \\
\frac{2}{a} \int_{H_{*}}^{H}\left[c-\frac{\gamma-1}{\gamma} H\left(\frac{d W}{d H}\right)^{2}\right] H^{-\gamma /(\gamma-1)} d H \\
+\frac{1}{a} \ln \left[1+\frac{a \tau}{\rho_{0} x_{0}}\right], & \alpha=-\frac{1}{2} ;\end{cases} \\
& \frac{t(H, \tau)}{t_{0}}=\left\{\begin{array}{ll}
\frac{\left(1+a \tau / \rho_{0} x_{0}\right)^{\alpha+1}}{a(x} \int_{H_{*}}^{H}\left[\frac{\gamma-1}{\gamma} H\left(\frac{d W}{d H}\right)^{2}-c\right] \frac{H^{-\gamma /(\gamma-1)}}{W} d H \\
+\frac{1}{a(\alpha+1)}\left[\left(1+\frac{a \tau}{\rho_{0} x_{0}}\right)^{\alpha+1}-1\right], \quad \alpha \neq-1, \\
\frac{1}{a} \int_{H_{*}}^{H}\left[c-\frac{\gamma-1}{\gamma} H\left(\frac{d W}{d H}\right)^{2}\right] \frac{H^{(-\gamma /(\gamma-1))}}{W} d H \\
+\frac{1}{a} \ln \left[1+\frac{a \tau}{\rho_{0} x_{0}}\right],
\end{array}\right]
\end{aligned}
$$

where $c=\frac{1}{2}\left(\frac{2}{\gamma+1}\right)^{(\gamma+1) / \gamma}, H_{*} \leq H<\infty$ if $a \alpha>0$, and $1<H \leq H_{*}$ if $a \alpha<0$. 
Setting $\tau=$ constant in (6.33)-(6.34), we get the trajectories of the gas particles or the lines of constant entropy in a parametric form. When $\tau=0$, these equations give the parametric representation of the law of motion of the piston:

$$
\begin{gathered}
\frac{x(H)}{x_{0}}=\frac{1}{a \alpha} \int_{H_{*}}^{H}\left[\frac{\gamma-1}{\gamma} H\left(\frac{d W}{d H}\right)^{2}-c\right] H^{-\gamma /(\gamma-1)} d H, \\
\frac{t(H)}{t_{0}}=\frac{1}{a \alpha} \int_{H_{*}}^{H}\left[\frac{\gamma-1}{\gamma} H\left(\frac{d W}{d H}\right)^{2}-c\right] \frac{H^{-\gamma /(\gamma-1)}}{W} d H .
\end{gathered}
$$

The solution (6.23)-(6.24), (6.12)-(6.13), (6.21)-(6.22), (6.30), and (6.35) of the piston problem posed in Sec. 4 involves four arbitrary constants $\rho_{0}, x_{0}, t_{0}$, and $\alpha$. The nature of these solutions is similar to those obtained in Sec. 4 .

It is easy to check that the series (6.23)-(6.24) with (6.12)-(6.13) and (6.21)-(6.22) can be summed up only when $\alpha=-1,-1 / 2$.

For $\alpha=-1$, Eqs. (6.21) $-(6.22)$ give

$$
\begin{aligned}
W_{*}^{(1)}= & \frac{\gamma}{\gamma-1}\left(H^{(0)}\right)^{1 /(\gamma-1)} H_{*}^{(1)}, \\
W_{*}^{(2)}= & \frac{\gamma}{\gamma-1}\left(H^{(0)}\right)^{1 /(\gamma-1)} H_{*}^{(2)}+\frac{\gamma}{(\gamma-1)^{2}}\left(H^{(0)}\right)^{(2-\gamma) /(\gamma-1)}\left(H_{*}^{(1)}\right)^{2}, \\
W_{*}^{(3)}= & \frac{\gamma}{\gamma-1}\left(H^{(0)}\right)^{1 /(\gamma-1)} H_{*}^{(3)}+\frac{3 \gamma}{(\gamma-1)^{2}}\left(H^{(0)}\right)^{(2-\gamma) /(\gamma-1)} H_{*}^{(1)} H_{*}^{(2)} \\
& +\frac{\gamma(2-\gamma)}{(\gamma-1)^{3}}\left(H^{(0)}\right)^{(1-2 \gamma) /(\gamma-1)}\left(H_{*}^{(1)}\right)^{3},
\end{aligned}
$$

etc., for $n=0,1, \ldots$, and

$$
W^{(0)}=\left(H^{(0)}\right)^{\gamma /(\gamma-1)}
$$

follows from (6.12)-(6.13). For the relations (6.36)-(6.39) between the coefficients of the series $(6.23)-(6.24)$, we find that

$$
W=H^{\gamma /(\gamma-1)} .
$$

Thus, for $\alpha=-1$, the solution may be written as

$$
\begin{gathered}
u=U H^{\gamma /(\gamma-1)}, \quad \rho_{*}=\rho_{0}\left(1-\frac{a x}{x_{0}}\right)^{-2}, \\
f=c_{*} \frac{x_{0}^{2}}{t_{0}^{2}}\left(1+\frac{a \tau}{\rho_{0} x_{0}}\right)^{-2}, \quad U=\frac{x_{0}}{t_{0}} \exp \left(-\frac{a t}{t_{0}}\right), \\
\frac{x(t)}{x_{0}}=\frac{1}{a}\left[1-\exp \left(-\frac{a t}{t_{0}}\right)\right], \\
\frac{x(H)}{x_{0}}=\frac{1}{a} \int_{H_{*}}^{H}\left[c-\frac{\gamma-1}{\gamma} H\left(\frac{d W}{d H}\right)^{2}\right] H^{-\gamma /(\gamma-1)} d H, \\
\frac{t(H)}{t_{0}}=\frac{1}{a} \int_{H_{*}}^{H}\left[c-\frac{\gamma-1}{\gamma} H\left(\frac{d W}{d H}\right)^{2}\right] \frac{H^{-\gamma /(\gamma-1)}}{W} d H,
\end{gathered}
$$


where $H_{*} \leq H<\infty$ if $a=-1$, and $1<H \leq H_{*}$ if $a=1$. This solution involves only three arbitrary constants $\rho_{0}, x_{0}$, and $t_{0}$.

For $\alpha=-1 / 2$, if we assume that the solution has the form

$$
W=(G(H))^{1 / 2},
$$

where

$$
W^{(0)}=\left(H^{(0)}\right)^{\gamma /(\gamma-1)} \text { or } G\left(H^{(0)}\right)=\left(H^{(0)}\right)^{2 \gamma /(\gamma-1)},
$$

and use the Taylor expansion of $G$ in (6.42) about $\sigma=0$, then in view of (6.22)(6.23), we get

$$
\begin{gathered}
\left(\frac{d G}{d H}\right)_{H=H^{(0)}}=-2 \gamma c+c_{1}\left(H^{(0)}\right)^{1 /(\gamma-1)}, \quad c_{1}=\frac{2 \gamma}{\gamma-1}, \\
\left(\frac{d^{2} G}{d H^{2}}\right)_{H=H^{(0)}}=\frac{c_{1}}{\gamma-1}\left(H^{(0)}\right)^{(2-\gamma) /(\gamma-1)}, \\
\left(\frac{d^{3} G}{d H^{3}}\right)_{H=H^{(0)}}=\frac{(2-\gamma) c_{1}}{(\gamma-1)^{2}}\left(H^{(0)}\right)^{(3-\gamma) /(\gamma-1)},
\end{gathered}
$$

etc., corresponding to $n=0,1, \ldots$. We may therefore conclude that

$$
G=-2 \gamma c H+2 H^{\gamma /(\gamma-1)}
$$

where $c$ is defined in (6.34).

Now, for $\alpha=-1 / 2$, the solution is

$$
\begin{gathered}
u=U W, \quad \rho_{*}=\rho_{0} \exp \left(\frac{a x}{x_{0}}\right), \\
U=\frac{x_{0}}{t_{0}}\left(1+\frac{a t}{2 t_{0}}\right)^{-1}, \quad f=c_{*} \frac{x_{0}^{2}}{t_{0}^{2}}\left(1+\frac{a \tau}{\rho_{0} x_{0}}\right)^{-1}, \\
\frac{x(t)}{x_{0}}=\frac{2}{a} \ln \left[1+\frac{a t}{2 t_{0}}\right], \\
\frac{x(H)}{x_{0}}=\frac{2}{a} \int_{H *}^{H}\left[c-\frac{\gamma-1}{\gamma} H\left(\frac{d W}{d H}\right)^{2}\right] H^{-i /(\gamma-1)} d H, \\
\frac{t(H)}{t_{0}}=\frac{2}{a} \int_{H_{*}}^{H}\left[c-\frac{\gamma-1}{\gamma} H\left(\frac{d W}{d H}\right)^{2}\right] \frac{H^{-\gamma /(\gamma-1)}}{W} d H,
\end{gathered}
$$

where

$$
W=\left[-2 \gamma c H+2 H^{i /(i-1)}\right]^{1 / 2} \text {. }
$$

(II) Exponential type similarity solutions of the system (6.2)-(6.3). We may now choose

$$
\begin{gathered}
\rho_{*}=\rho_{0} \exp \left(-\frac{2 a \tau}{\rho_{0} x_{0}}\right), \\
U=\frac{x_{0}}{t_{0}} \exp \left(\frac{a \tau}{\rho_{0} x_{0}}\right), \\
f=c_{*} \frac{x_{0}^{2}}{t_{0}^{2}} \exp \left(\frac{2 a \tau}{\rho_{0} x_{0}}\right),
\end{gathered}
$$


where $\rho_{0}, x_{0}$, and $t_{0}$ are arbitrary (dimensional) constants, and $c_{*}$ is given by (6.17).

Substituting (6.49)-(6.51) into (6.9)-(6.10) we find that the first and higher order coefficients in the series (6.7)-(6.8) are given by

$$
\begin{aligned}
& W^{(n+1)}(z)=W_{*}^{(n+1)} z^{n+1}, \\
& H^{(n+1)}(z)=H_{*}^{(n+1)} z^{n+1}, \quad n=0,1, \ldots,
\end{aligned}
$$

where

$$
z=\frac{t_{0}}{\rho_{0} x_{0}^{2}} \exp \left(-\frac{a \tau}{\rho_{0} x_{0}}\right)
$$

and

$$
\begin{aligned}
& W_{*}^{(n+1)}=\frac{\gamma}{(\gamma-1) H^{(0)}}\left[W^{(0)} H_{*}^{(n+1)}-a n H_{*}^{(n)}+T_{*}^{(n)}\left(\left\{W_{*}^{(i)}\right\},\left\{H_{*}^{(i)}\right\}\right)\right], \\
& H_{*}^{(n+1)}=\frac{1}{D_{*}}\left[a(1-n) W_{*}^{(n)}-\frac{a n \gamma W^{(0)}}{(\gamma-1) H^{(0)}} H_{*}^{(n)}+S_{*}^{(n)}\left(\left\{W_{*}^{(i)}\right\},\left\{H_{*}^{(i)}\right\}\right)\right]
\end{aligned}
$$

are constant with

$$
\begin{aligned}
T_{*}^{(n)}\left(\left\{W_{*}^{(i)}\right\},\left\{H_{*}^{(i)}\right\}\right) & =\sum_{k=1}^{n}\left(\begin{array}{l}
n \\
k
\end{array}\right)\left[W_{*}^{(k)} H_{*}^{(n+1-k)}-\frac{\gamma-1}{\gamma} H_{*}^{(k)} W_{*}^{(n+1-k)}\right], \\
S_{*}^{(n)}\left(\left\{W_{*}^{(i)}\right\},\left\{H_{*}^{(i)}\right\}\right) & =\sum_{k=1}^{n}\left(\begin{array}{l}
n \\
k
\end{array}\right) W_{*}^{(k)} W_{*}^{(n+1-k)}+\frac{\gamma W^{(0)}}{(\gamma-1) H^{(0)}} T^{(n)}\left(\left\{W_{*}^{(i)}\right\},\left\{H_{*}^{(i)}\right\}\right), \\
D_{*} & =\frac{1}{\gamma-1}\left(\frac{2}{\gamma+1}\right)^{1 / \gamma} ;
\end{aligned}
$$

$W^{(0)}$ and $H^{(0)}$ are given by (6.12)-(6.13). Again, we may rewrite the series (6.7)(6.8), using (6.12)-(6.13) and (6.52)-(6.56), as

$$
\begin{gathered}
W=\sum_{j=0}^{\infty} W_{*}^{(j)} \frac{\sigma^{j}}{j !}, \\
H=\sum_{j=0}^{\infty} H_{*}^{(j)} \frac{\sigma^{j}}{j !},
\end{gathered}
$$

where

$$
\sigma=\frac{t_{0} s}{\rho_{0} x_{0}^{2}} \exp \left(-\frac{a \tau}{\rho_{0} x_{0}}\right)
$$

It may be checked that (6.57)-(6.58) with (6.12)-(6.13) and (6.55)-(6.56) is also the solution of the system of the ODE

$$
\begin{gathered}
(W-a \sigma) \frac{d H}{d \sigma}-\frac{\gamma-1}{\gamma} H \frac{d W}{d \sigma}=0, \\
(W-a \sigma) \frac{d W}{d \sigma}-c_{*} b_{0}^{(\gamma-1) / \gamma} \frac{d H}{d \sigma}+a W=0,
\end{gathered}
$$


with initial conditions (6.5)-(6.6) at the shock. The ODE (6.60)-(6.61) may be obtained from (6.2)-(6.3) by using the similarity variable (6.59); these are not soluble in a closed form.

The parametric representation of shock may be found to be

$$
\begin{gathered}
\frac{x}{x_{0}}=\frac{1}{2 a}\left[\exp \left(\frac{2 a \tau}{\rho_{0} x_{0}}\right)-1\right], \\
\frac{t}{t_{0}}=\frac{1}{a}\left[\exp \left(\frac{a \tau}{\rho_{0} x_{0}}\right)-1\right]
\end{gathered}
$$

(see Eqs. (2.11), (3.2), and (6.49)-(6.50)). The shock trajectory in the $(x, t)$-plane, shock velocity, and undisturbed density have the form

$$
\begin{gathered}
\frac{x(t)}{x_{0}}=\frac{1}{2 a}\left[\left(1+\frac{a t}{t_{0}}\right)^{2}-1\right], \\
U=\frac{x_{0}}{t_{0}}\left(1+\frac{a t}{t_{0}}\right), \\
\rho_{*}=\rho_{0}\left(1+\frac{2 a x}{x_{0}}\right)^{-1} .
\end{gathered}
$$

The functions $x(H, \tau)$ and $t(H, \tau)$ are given by

$$
\begin{aligned}
\frac{x(H, \tau)}{x_{0}}= & \frac{\exp \left(2 a \tau / \rho_{0} x_{0}\right)}{a} \int_{H_{*}}^{H}\left[\frac{\gamma-1}{\gamma} H\left(\frac{d W}{d H}\right)^{2}-c\right] H^{-\gamma /(\gamma-1)} d H \\
& +\frac{1}{2 a}\left[\exp \left(\frac{2 a \tau}{\rho_{0} x_{0}}\right)-1\right], \\
\frac{t(H, \tau)}{t_{0}}= & \frac{\exp \left(a \tau / \rho_{0} x_{0}\right)}{a} \int_{H .}^{H}\left[\frac{\gamma-1}{\gamma} H\left(\frac{d W}{d H}\right)^{2}-c\right] \frac{H^{-\gamma /(\gamma-1)}}{W} d H \\
& +\frac{1}{a}\left[\exp \left(\frac{a \tau}{\rho_{0} x_{0}}\right)-1\right],
\end{aligned}
$$

where $c=\frac{1}{2}\left(\frac{2}{\gamma+1}\right)^{(\gamma+1) / \gamma}, H_{*} \leq H<\propto$ if $a=1$, and $1<H \leq H_{*}$ if $a=-1$.

Equations (6.67)-(6.68), with $\tau=$ constant, give the trajectories of the gas particles. When $\tau=0$ we get the parametric representation of the piston path as

$$
\begin{gathered}
\frac{x(H)}{x_{0}}=\frac{1}{a} \int_{H_{.}}^{H}\left[\frac{\gamma-1}{\gamma} H\left(\frac{d W}{d H}\right)^{2}-c\right] H^{-\gamma /(\gamma-1)} d H, \\
\frac{t(H)}{t_{0}}=\frac{1}{a} \int_{H_{.}}^{H}\left[\frac{\gamma-1}{\gamma} H\left(\frac{d W}{d H}\right)^{2}-c\right] \frac{H^{-\gamma /(\gamma-1)}}{W} d H .
\end{gathered}
$$

Piston path and shock locus for $b_{0}=5 / 3, \gamma=7 / 5$, and $a=1$ are shown in Fig. 3 . In this case, the undisturbed density vanishes in an infinite distance, and shock velocity becomes infinite in an infinite time. 


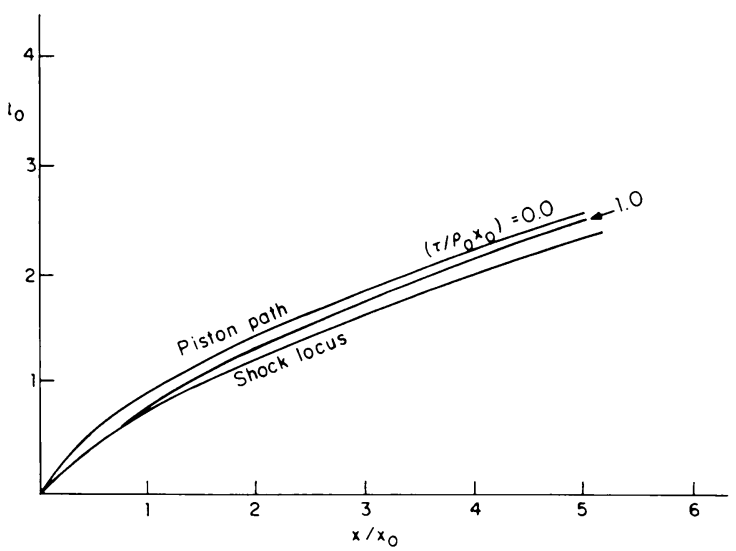

FIG. 3. Piston path, particle trajectory, and shock locus with $a=1, \gamma=7 / 5, b_{0}=5 / 3$

(III) Strong shocks produced by an impulsive piston motion. Now we consider gas flows arising from the short intense impact of a piston moving under gas pressure. For this we introduce the coordinates $y$ and $\beta$ according to

$$
\begin{gathered}
d \tau=m d y, \\
d \xi=d s=-m a_{0} d \beta,
\end{gathered}
$$

where $\tau$ and $s$ (or $\xi$ ) are given by (3.1) and (3.2), respectively; $m$ and $a_{0}$ are positive constants with the dimensions $M L^{-2}$ and $L T^{-1}$, respectively. The shock is now given by $\beta=0$ while the piston path is identified by $y=0$.

With $\beta$ and $y$ as independent variables, Eqs. (3.23)-(3.24) become

$$
\begin{gathered}
W_{y}+W W_{\beta}-f H_{\beta}=0, \\
H_{y}+W H_{\beta}-\frac{\gamma-1}{\gamma} H W_{\beta}=0,
\end{gathered}
$$

where

$$
W=-\frac{u}{a_{0}}, \quad H=\frac{p^{(y-1) / \gamma}}{a_{0}^{2}}
$$

and $f$ is given by (3.25).

We seek exact solutions of (6.72)-(6.73) in the form

$$
\begin{gathered}
W(y, \beta)=W^{(0)}(y)+2 W^{(1)}(y) \beta, \\
H(y, \beta)=H^{(0)}(y)+H^{(1)}(y) \beta+H^{(2)}(y) \beta^{2} .
\end{gathered}
$$

In (6.75)-(6.76) if $W^{(0)}(0)=-1$ and $W^{(1)}(0)=1 / 2$, then the solution (6.75)(6.76) corresponds to the gas flow in the region between a strong shock and a piston, produced by the motion of a piston after a brief impact. As a result, the piston, which is of mass $m$ per unit cross-sectional area, instantaneously acquires a finite velocity $a_{0}>0$ and for $t>0$ moves under the influence of gas pressure [3]. Consequently,

$$
\begin{array}{ll}
W(y, 0)=0, & y \geq 0, \\
W(0, \beta)=\beta-1, & \beta \geq 0 .
\end{array}
$$


Substituting (6.75)-(6.76) into (6.72) and equating the coefficients of different powers of $\beta$ to zero, we have

$$
\begin{gathered}
\frac{d W^{(0)}}{d y}+2 W^{(0)} W^{(1)}-f H^{(1)}=0 \\
\frac{d W^{(1)}}{d y}+2\left(W^{(1)}\right)^{2}-f H^{(2)}=0
\end{gathered}
$$

Similarly, Eq. (6.73) yields

$$
\begin{gathered}
\frac{d H^{(0)}}{d y}+W^{(0)} H^{(1)}-\frac{2(\gamma-1)}{y} H^{(0)} W^{(1)}=0, \\
\frac{d H^{(1)}}{d y}+2\left(W^{(0)} H^{(2)}+W^{(1)} H^{(1)}\right)-\frac{2(\gamma-1)}{\gamma} H^{(1)} W^{(1)}=0, \\
\frac{d H^{(2)}}{d y}+4 W^{(1)} H^{(2)}-\frac{2(\gamma-1)}{\gamma} H^{(2)} W^{(1)}=0 .
\end{gathered}
$$

The shock conditions (2.12)-(2.14) give

$$
\begin{aligned}
& H_{\beta=0}=\frac{1}{2 f}\left(W_{\beta=0}\right)^{2}, \\
& U=-\frac{\gamma+1}{2} a_{0} W_{\beta=0},
\end{aligned}
$$

where $W_{\beta=0}$ is a function of $y$ to be determined. Equations (6.78)-(6.79) give

$$
\begin{aligned}
& H^{(1)}=\frac{1}{f}\left[\frac{d W^{(0)}}{d y}+2 W^{(0)} W^{(1)}\right], \\
& H^{(2)}=\frac{1}{f}\left[\frac{d W^{(1)}}{d y}+2\left(W^{(1)}\right)^{2}\right] .
\end{aligned}
$$

Equations (6.75)-(6.76) show that

$$
W^{(0)}=W_{\beta=0}, \quad H^{(0)}=H_{\beta=0} .
$$

Using (6.83), (6.85), and (6.87) in (6.80), we get

$$
\frac{1}{f} \frac{d f}{d y}=\frac{4}{W^{(0)}} \frac{d W^{(0)}}{d y}+\frac{2(\gamma+1)}{\gamma} W^{(1)} .
$$

In view of (3.25), Eq. (6.88) may be rewritten as

$$
\frac{1}{\rho_{*}} \frac{d \rho_{*}}{d y}=\frac{2(1-2 \gamma)}{(\gamma-1) W^{(0)}} \frac{d W^{(0)}}{d y}-\frac{2(\gamma+1)}{\gamma-1} W^{(1)} .
$$

Equations (6.79), (6.81), and (6.88) yield

$$
\frac{d^{2} W^{(0)}}{d y^{2}}+4 W^{(0)} \frac{d W^{(1)}}{d y}-\frac{4}{W^{(0)}}\left(\frac{d W^{(0)}}{d y}\right)^{2}-8 W^{(1)} \frac{d W^{(0)}}{d y}=0,
$$


and (6.79) and (6.82), after an integration, give

$$
\frac{d W^{(1)}}{d y}+2 W^{(1)^{2}}=c\left(W^{(0)}\right)^{2}
$$

where $c$ is a constant of integration.

We try to find a solution of ODE (6.90)-(6.91) in the form

$$
\begin{aligned}
& W^{(0)}(y)=\sum_{i=0}^{\infty} W_{i}^{(0)} y^{i}, \\
& W^{(1)}(y)=\sum_{i=0}^{\infty} W_{i}^{(1)} y^{i},
\end{aligned}
$$

where $W_{i}^{(0)}$ and $W_{i}^{(1)}, i \geq 0$, are constant. The substitution of (6.92)-(6.93) into (6.90)-(6.91) leads to the following algebraic relations for $W_{i}^{(0)}$ and $W_{i}^{(1)}$, with $i=n+1$ :

$$
\begin{aligned}
& W_{n+2}^{(0)}=\frac{1}{(n+1)(n+2) W_{0}^{(0)}[4\{} \sum_{k=0}^{n}(k+1)(n-k+1) W_{k+1}^{(0)} W_{n+1-k}^{(0)} \\
&+2 \sum_{k=0}^{n} \sum_{p=0}^{n-k}(k+1) W_{p}^{(0)} W_{k+1}^{(0)} W_{n-k-p}^{(1)} \\
&\left.-\sum_{p=0}^{n} \sum_{k=0}^{n-p}(p+q) W_{k}^{(0)} W_{n-k-p}^{(0)} W_{p+1}^{(1)}\right\} \\
&\left.-\sum_{k=0}^{n-1}(k+1)(k+2) W_{k+2}^{(0)} W_{n-k}^{(0)}\right], \\
& W_{n+1}^{(1)}=\frac{1}{(n+1)}\left[c \sum_{k=0}^{n} \sum_{p=0}^{k} \sum_{q=0}^{n-k} W_{p}^{(0)} W_{q}^{(0)} W_{k-p}^{(0)} W_{n-k-p}^{(0)}-2 \sum_{p=0}^{n} W_{p}^{(1)} W_{n-p}^{(1)}\right],
\end{aligned}
$$

$n=0,1, \ldots$. Thus, $(6.92)-(6.93)$ with $(6.94)-(6.95)$ is the series solution of ODE (6.90)-(6.91), involving three arbitrary constants $W_{0}^{(0)}, W_{0}^{(1)}$, and $W_{1}^{(0)}$. These series can be summed up only when $c=0$, as we now show. This special case was considered by Ustinov [3].

When $c=0$, Eq. (6.95) gives

$$
\begin{aligned}
& W_{1}^{(1)}=-2\left(W_{0}^{(1)}\right)^{2}, \\
& W_{2}^{(1)}=-2 W_{0}^{(1)} W_{1}^{(1)}, \\
& W_{3}^{(1)}=-\frac{2}{3}\left[\left(W^{(1)}\right)^{2}+2 W_{0}^{(1)} W_{2}^{(1)}\right],
\end{aligned}
$$

etc., and in view of (6.77), we have

$$
W_{0}^{(1)}=\frac{1}{2}, \quad W_{1}^{(1)}=-\frac{1}{2}, \quad W_{2}^{(1)}=\frac{1}{2}, \quad W_{3}^{(1)}=-\frac{1}{2},
$$

etc. The series (6.93) can now be summed up to give

$$
W^{(1)}(y)=\frac{1}{2}(1+y)^{-1} .
$$


Similarly, we have

$$
W_{0}^{(0)}=-1
$$

from (6.77), and if we assume that

$$
W_{1}^{(0)}=-\frac{\left(c_{1}+2\right)}{3},
$$

where $c_{1}$ is an arbitrary constant, Eq. (6.94) yields

$$
\begin{aligned}
& W_{2}^{(0)}=-\left(5+2 c_{1}^{2}+2 c_{1}\right) / g, \\
& W_{3}^{(0)}=-\left(14 c_{1}^{3}+12 c_{1}^{2}+7 c_{1}+40\right) / 81,
\end{aligned}
$$

etc. Thus, the series (6.92) can also be summed up to give

$$
W^{(0)}(y)=-\left(1+c_{1} y\right)^{-1 / 3}(1+y)^{-2 / 3} .
$$

Substituting $W^{(0)}$ and $W^{(1)}$ from (6.104) and (6.99) into (6.89) and integrating, we get

$$
\rho_{*}=\rho_{0}\left(1+c_{1} y\right)^{2(2 \gamma-1) / 3(\gamma-1)}(1+y)^{(5 y-7) / 3(\gamma-1)},
$$

where $\rho_{0}$ is the density of the gas at the piston.

The particle velocity in the region between the shock wave and the piston is now given by

$$
u=a_{0}\left[\left(1+c_{1} y\right)^{-1 / 3}(1+y)^{-2 / 3}+(1+y)^{-1} \beta\right]
$$

(see (6.74)). The law of motion of the shock wave in parametric form is found to be

$$
\begin{aligned}
x & =\frac{m}{\rho_{0}} \int_{0}^{y}\left(1+c_{1} y\right)^{2(1-2 \gamma) / 3(\gamma-1)}(1+y)^{-(5 \gamma-7) / 3(\gamma-1)} d y, \\
t & =\frac{2 m}{(\gamma+1) a_{0} \rho_{0}} \int_{0}^{y}\left(1+c_{1} y\right)^{(1-3 \gamma) / 3(\gamma-1)}(1+y)^{(5-3 \gamma) / 3(\gamma-1)} d y .
\end{aligned}
$$

A detailed discussion of the gas flows described by the above special solution may be found in [3] for the cases $c_{1}<0$ and $c_{1}=0$. Ustinov also noted that when $c \neq 0$ in (6.91), Eqs. (6.90)-(6.91) may be transformed to an Abel equation of the second kind:

$$
\frac{d \mu}{d \sigma}=\frac{(3 \sigma-14) \mu^{2}+16 \sigma \mu+4 \sigma^{2}(\sigma-2)}{4 \sigma \mu(\sigma-2)},
$$

where

$$
\sigma=\left(W^{(1)}\right)^{-1} \frac{d W^{(1)}}{d y}+2, \quad \mu=W^{(1)} \frac{d \sigma}{d W^{(1)}}, \quad\left(W^{(0)}\right)^{4}=\frac{1}{c} \sigma\left(W^{(1)}\right)^{2} .
$$

The solution of Eqs. (6.108)-(6.109) may again be obtained in the form

$$
\begin{aligned}
& W^{(0)}(y)=\sum_{i=0}^{\infty} W_{i}^{(0)} y^{i}, \\
& W^{(1)}(y)=\sum_{i=0}^{\infty} W_{i}^{(1)} y^{i},
\end{aligned}
$$




$$
\begin{aligned}
& \mu(y)=\sum_{i=0}^{\infty} \mu_{i} y^{i}, \\
& \sigma(y)=\sum_{i=0}^{\infty} \sigma_{i} y^{i},
\end{aligned}
$$

where $W_{i}^{(0)}, W_{i}^{(1)}, \mu_{i}$, and $\sigma_{i}, i \geq 0$, are constant. The constant coefficients in the series (6.110)-(6.113) can be determined recursively through their algebraic dependence on lower-order coefficients. The convergence of the series solution (6.110)(6.113) can be checked numerically.

7. Solutions of the system (3.27)-(3.28) with strong shocks. If we introduce

$$
W=\frac{u^{2}}{\phi^{2}}, \quad H=\left(\frac{p}{b_{0}}\right)^{(\gamma-1) / \gamma}
$$

as new dependent variables, Eqs. (3.27)-(3.28) become

$$
\begin{gathered}
H_{\tau}+\left(\frac{1}{2} W \phi^{2}+f b_{0}^{(\gamma-1) / \gamma} H\right) H_{s}-\frac{\gamma-1}{2 \gamma} \phi^{2} H W_{s}=0, \\
\phi\left[W_{\tau}+\left(\frac{1}{2} W \phi^{2}+f b_{0}^{(\gamma-1) / \gamma} H\right) W_{s}\right]-2 f b_{0}^{(\gamma-1) / \gamma} \phi W H_{s}+2 W \frac{d \phi}{d \tau}=0,
\end{gathered}
$$

where $\phi(\tau)$ is an arbitrary function of $\tau$ and $b_{0}$ is an arbitrary constant with the dimension of pressure. We assume that the solution of Eqs. (7.2)-(7.3) has the form

$$
\begin{aligned}
& W(\tau, s)=\sum_{j=0}^{\infty} W^{(j)}(\tau) \frac{s^{j}}{j !}, \\
& H(\tau, s)=\sum_{j=0}^{\infty} H^{(j)}(\tau) \frac{s^{j}}{j !} .
\end{aligned}
$$

We substitute (7.4)-(7.5) into Eqs. (7.2)-(7.3) and equate the coefficients of different powers of $s$ to zero. This leads to the following algebraic relations for $W^{(j)}$ and $H^{(j)}, j \geq 1$ :

$$
\begin{array}{r}
W^{(n+1)}(\tau)=\frac{2 \gamma}{(\gamma-1) \phi^{2} H^{(0)}}\left[\left(\frac{1}{2} W^{(0)} \phi^{2}+f b_{0}^{(\gamma-1) / \gamma} H^{(0)}\right) H^{(n+1)}+\frac{d H^{(n)}}{d \tau}\right. \\
\left.+T^{(n)}\left(\left\{W^{(i)}\right\},\left\{H^{(i)}\right\}\right)\right], \\
H^{(n+1)}(\tau)=\frac{1}{D_{0}}\left[W^{(n)} \frac{d \phi}{d \tau}+\frac{\phi}{2} \frac{d W^{(n)}}{d \tau}+\frac{\gamma}{(\gamma-1) \phi H^{(0)}}\right. \\
\left.\cdot\left(\frac{1}{2} W^{(0)} \phi^{2}+f b_{0}^{(\gamma-1) / \gamma} H^{(0)}\right) \frac{d H^{(n)}}{d \tau}+S^{(n)}\left(\left\{W^{(i)}\right\},\left\{H^{(i)}\right\}\right)\right],
\end{array}
$$


where

$$
\begin{aligned}
T^{(n)}\left(\left\{W^{(i)}\right\},\left\{H^{(i)}\right\}\right)= & \sum_{k=1}^{n}\left(\begin{array}{l}
n \\
k
\end{array}\right)\left[\left(\frac{1}{2} \phi^{2} W^{(k)}+f b_{0}^{(\gamma-1) / \gamma} H^{(k)}\right) H^{(n+1-k)}\right. \\
& \left.-\frac{\gamma-1}{2 \gamma} \phi^{2} H^{(k)} W^{(n+1-k)}\right]
\end{aligned}
$$

$$
\begin{aligned}
& S^{(n)}\left(\left\{W^{(i)}\right\},\left\{H^{(i)}\right\}\right) \\
& \quad=\frac{\phi}{2} \sum_{k=1}^{n}\left(\begin{array}{l}
n \\
k
\end{array}\right)\left[\left(\frac{1}{2} W^{(k)} \phi^{2}+f b_{0}^{(\gamma-1) / \gamma} H^{(k)}\right) W^{(n+1-k)}-2 f b_{0}^{(\gamma-1) / \gamma} W^{(k)} H^{(n+1-k)}\right] \\
& \quad+\left(\frac{\gamma}{\gamma-1}\right)\left(\phi H^{(0)}\right)^{-1}\left(\frac{1}{2} W^{(0)} \phi^{2}+f b_{0}^{(\gamma-1) / \gamma} H^{(0)}\right) T^{(n)}\left(\left\{W^{(i)}\right\},\left\{H^{(i)}\right\}\right), \\
& \quad D_{0}=f b_{0}^{(\gamma-1) / \gamma} \phi W^{(0)}-\left(\frac{\gamma}{(\gamma-1) \phi H^{(0)}}\right)\left(\frac{1}{2} W^{(0)} \phi^{2}+f b_{0}^{(\gamma-1) / \gamma} H^{(0)}\right)^{2} .
\end{aligned}
$$

If we choose

$$
\phi=U, \quad \rho_{*} U^{2}=b_{0}=\frac{\rho_{0} x_{0}^{2}}{t_{0}^{2}},
$$

where $U$ is the shock velocity, Eqs. (2.12)-(2.14) give

$$
\begin{aligned}
& W_{s=0}=\left(\frac{2}{\gamma+1}\right)^{2}, \\
& H_{s=0}=\left(\frac{2}{\gamma+1}\right)^{(\gamma-1) / \gamma} .
\end{aligned}
$$

Thus, the basic solution in the series (7.4)-(7.5) is given, respectively, by (7.10) and (7.11). The series solution (7.4)-(7.5), (7.10)-(7.11), and (7.6)-(7.8) of the PDE (7.2)-(7.3) involves two arbitrary functions, namely, $\phi$ and $f$. These arbitrary functions may be suitably chosen to derive power-law and exponential type similarity solutions.

(I) Power-law similarity solutions of the system (7.2)-(7.3). To derive the powerlaw similarity solutions, we choose

$$
\begin{aligned}
& \rho_{*}=\rho_{0}\left(1+\frac{a \tau}{\rho_{0} x_{0}}\right)^{-\alpha}, \\
& U=\frac{x_{0}}{t_{0}}\left(1+\frac{a \tau}{\rho_{0} x_{0}}\right)^{\alpha / 2}, \\
& f=c_{*} \frac{x_{0}^{2}}{t_{0}^{2}}\left(1+\frac{a \tau}{\rho_{0} x_{0}}\right)^{\alpha},
\end{aligned}
$$

where $x_{0}, t_{0}, \rho_{0}, a$, and $\alpha$ are the same as defined in Sec. 6, and the parameter $c_{*}$ is given by (6.17). 
Using (7.12)-(7.14) in (7.6)-(7.8), we have

$$
\begin{aligned}
& W^{(n+1)}(z)=W_{*}^{(n+1)} z^{n+1}, \\
& H^{(n+1)}(z)=H_{*}^{(n+1)} z^{n+1}, \quad n=0,1,2, \ldots,
\end{aligned}
$$

where

and

$$
z=\frac{t_{0}^{2}}{\rho_{0} x_{0}^{3}}\left(1+\frac{a \tau}{\rho_{0} x_{0}}\right)^{-(\alpha+1)}
$$

$$
\begin{aligned}
& W_{*}^{(n+1)}=\frac{2 \gamma}{(\gamma-1) H^{(0)}}\left[\left(\frac{1}{2} W^{(0)}+c H^{(0)}\right) H_{*}^{(n+1)}-\right. \operatorname{an}(\alpha+1) H_{*}^{(n)} \\
&\left.+T_{*}^{(n)}\left(\left\{W_{*}^{(i)}\right\},\left\{H_{*}^{(i)}\right\}\right)\right], \\
& H_{*}^{(n+1)}=\frac{1}{D_{*}}\left[(\alpha-(\alpha+1) n) a W_{*}^{(n)}-\frac{2 \gamma a n(\alpha+1)}{(\gamma-1) H^{(0)}}\left(\frac{1}{2} W^{(0)}+c H^{(0)}\right) H_{*}^{(n)}\right. \\
&\left.+S_{*}^{(n)}\left(\left\{W_{*}^{(i)}\right\},\left\{H_{*}^{(i)}\right\}\right)\right]
\end{aligned}
$$

are constants with

$$
\begin{aligned}
& T_{*}^{(n)}\left(\left\{W_{*}^{(i)}\right\},\left\{H_{*}^{(i)}\right\}\right) \\
&=\sum_{k=1}^{n}\left(\begin{array}{l}
n \\
k
\end{array}\right)\left[\left(\frac{1}{2} W_{*}^{(k)}+c H_{*}^{(k)}\right) H_{*}^{(n+1-k)}-\frac{\gamma-1}{2 \gamma} H_{*}^{(k)} W_{*}^{(n+1-k)}\right], \\
& S_{*}^{(n)}\left(\left\{W_{*}^{(i)}\right\},\left\{H_{*}^{(i)}\right\}\right)= \sum_{k=1}^{n}\left(\begin{array}{l}
n \\
k
\end{array}\right)\left[\left(\frac{1}{2} W_{*}^{(k)}+c H_{*}^{(k)}\right) W_{*}^{(n+1-k)}-2 c W_{*}^{(k)} H_{*}^{(n+1-k)}\right] \\
&+\frac{2 \gamma\left((1 / 2) W^{(0)}+c H^{(0)}\right)}{(\gamma-1) H^{(0)}} T_{*}^{(n)}\left(\left\{W_{*}^{(i)}\right\},\left\{H_{*}^{(i)}\right\}\right), \\
& D_{*}= 2 c W^{(0)}-\frac{2 \gamma}{(\gamma-1) H^{(0)}}\left(\frac{1}{2} W^{(0)}+c H^{(0)}\right)^{2}, \\
& c= \frac{1}{2}\left(\frac{2}{\gamma+1}\right)^{(\gamma+1) / \gamma} \cdot
\end{aligned}
$$

In view of (7.15)-(7.19), we may rewrite the series (7.4)-(7.5), as

$$
\begin{gathered}
W=\sum_{j=0}^{\infty} W_{*}^{(j)} \frac{\sigma^{j}}{j !}, \\
H=\sum_{j=0}^{\infty} H_{*}^{(j)} \frac{\sigma^{j}}{j !},
\end{gathered}
$$

where

$$
\sigma=\frac{s t_{0}^{2}}{\rho_{0} x_{0}^{3}}\left(1+\frac{a \tau}{\rho_{0} x_{0}}\right)^{-(\alpha+1)} .
$$


The solution series (7.20)-(7.21) with (7.10)-(7.11) and (7.18)-(7.19) may also be found to satisfy the ODE

$$
\begin{gathered}
{\left[-a(\alpha+1) \sigma+\frac{1}{2} W+c H\right] \frac{d H}{d \sigma}-\frac{(\gamma-1)}{2 \gamma} H \frac{d W}{d \sigma}=0,} \\
{\left[-a(\alpha+1) \sigma+\frac{1}{2} W+c H\right] \frac{d W}{d \sigma}-2 c W \frac{d H}{d \sigma}+\alpha a W=0,}
\end{gathered}
$$

which may be obtained from (7.2)-(7.3) by using the similarity of the variable $\sigma$ defined by (7.22).

The parametric representation of the shock now is

$$
\begin{array}{ll}
\frac{x}{x_{0}}= \begin{cases}\frac{1}{a(\alpha+1)}\left[\left(1+\frac{a \tau}{\rho_{0} x_{0}}\right)^{\alpha+1}-1\right], & \alpha \neq-1, \\
\frac{1}{a} \ln \left[1+\frac{a \tau}{\rho_{0} x_{0}}\right], & \alpha=-1 ;\end{cases} \\
\frac{t}{t_{0}}= \begin{cases}\frac{2}{a(\alpha+2)}\left[\left(1+\frac{a \tau}{\rho_{0} x_{0}}\right)^{(\alpha+2) / 2}-1\right], & \alpha \neq-2, \\
\frac{1}{a} \ln \left[1+\frac{a \tau}{\rho_{0} x_{0}}\right], & \alpha=-2 .\end{cases}
\end{array}
$$

The trajectory of the shock, shock velocity, and the density of the undisturbed gas are given, respectively, by

$$
\begin{aligned}
& \frac{x}{x_{0}}= \begin{cases}\frac{1}{a(\alpha+1)}\left[\left(1+\frac{a(\alpha+2)}{2 t_{0}} t\right)^{2(\alpha+1) /(\alpha+2)}-1\right], & \alpha \neq-1,-2, \\
\frac{2}{a} \ln \left[1+\frac{a t}{2 t_{0}}\right], & \alpha=-1, \\
\frac{1}{a}\left[1-\exp \left(-\frac{a t}{t_{0}}\right)\right], & \alpha=-2 ;\end{cases} \\
& U= \begin{cases}\frac{x_{0}}{t_{0}}\left[1+\frac{(\alpha+2) a t}{2 t_{0}}\right]^{\alpha /(\alpha+2)}, & \alpha \neq-2, \\
\frac{x_{0}}{t_{0}} \exp \left(-\frac{a t}{t_{0}}\right), & \alpha=-2\end{cases} \\
& \rho_{*}= \begin{cases}\rho_{0}\left[1+\frac{(\alpha+1) a x}{x_{0}}\right]^{-\alpha /(\alpha+1)}, & \alpha \neq-1, \\
\rho_{0} \exp \left(\frac{a x}{x_{0}}\right), & \alpha=-1 .\end{cases}
\end{aligned}
$$


The functions $x(H, \tau)$ and $t(H, \tau)$ are given by

$$
\begin{aligned}
& \frac{x(H, \tau)}{x_{0}}= \begin{cases}\frac{\left[1+a \tau / \rho_{0} x_{0}\right]^{\alpha+1}}{a \alpha} \int_{H_{*}}^{H}\left[\frac{\gamma-1}{2 \gamma} H\left(\frac{d W}{d H}\right)^{2}-2 c W\right] \frac{H^{-\gamma /(\gamma-1)}}{W} d H \\
\quad+\frac{\left[\left(1+a \tau / \rho_{0} x_{0}\right)^{\alpha+1}-1\right]}{a(\alpha+1)}, \quad \alpha \neq-1, \\
\frac{1}{a} \int_{H_{*}}^{H}\left[2 c W-\frac{\gamma-1}{2 \gamma} H\left(\frac{d W}{d H}\right)^{2}\right] \frac{H^{-\gamma /(\gamma-1)}}{W} d H \\
+\frac{1}{a} \ln \left[1+\frac{a \tau}{\rho_{0} x_{0}}\right], & \alpha=-1 ;\end{cases} \\
& \frac{t(H, \tau)}{t_{0}}=\left\{\begin{array}{c}
\frac{\left[1+a \tau / \rho_{0} x_{0}\right]^{(\alpha+2) / 2}}{a \alpha} \int_{H_{*}}^{H}\left[\frac{\gamma-1}{2 \gamma} H\left(\frac{d W}{d H}\right)^{2}-2 c W\right] \frac{H^{-\gamma /(\gamma-1)}}{W^{3 / 2}} d H \\
+\frac{2}{a(\alpha+2)}\left[\left(1+\frac{a \tau}{\rho_{0} x_{0}}\right)^{(\alpha+2) / 2}-1\right], \quad \alpha \neq-2, \\
\frac{1}{2 a} \int_{H_{*}}^{H}\left[2 c W-\frac{\gamma-1}{2 \gamma} H\left(\frac{d W}{d H}\right)^{2}\right] \frac{H^{-\gamma /(\gamma-1)}}{W^{3 / 2}} d H \\
+\frac{1}{a} \ln \left[1+\frac{a \tau}{\rho_{0} x_{0}}\right], \\
\alpha=-2 .
\end{array}\right.
\end{aligned}
$$

Here, $H_{*}=H_{s=0}$. The parametric representation of the piston motion is, therefore, given by

$$
\begin{aligned}
& \frac{x(H)}{x_{0}}=\frac{1}{a \alpha} \int_{H_{*}}^{H}\left[\frac{\gamma-1}{2 \gamma} H\left(\frac{d W}{d H}\right)^{2}-2 c W\right] \frac{H^{-\gamma /(\gamma-1)}}{W} d H, \\
& \frac{t(H)}{t_{0}}=\frac{1}{a \alpha} \int_{H_{*}}^{H}\left[\frac{\gamma-1}{2 \gamma} H\left(\frac{d W}{d H}\right)^{2}-2 c W\right] \frac{H^{-\gamma /(\gamma-1)}}{W^{3 / 2}} d H,
\end{aligned}
$$

where $H_{*} \leq H<\infty$ if $a \alpha>0$, and $1<H \leq H_{*}$ if $a \alpha<0$.

The above solution involves four arbitrary constants $x_{0}, t_{0}, \rho_{0}$, and $\alpha$. The following points may be noted from (7.28)-(7.29). The qualitative behavior of these solutions is similar to those discussed in Sec. 5 .

We find that for $\alpha=-1$, the series (7.20)-(7.21), using (7.10)-(7.11) and (7.18)(7.19), can be summed up. In this case, the soluiion has the form

$$
\begin{gathered}
u=U W^{1 / 2}, \quad \rho_{*}=\rho_{0} \exp \left(\frac{a x}{x_{0}}\right), \\
U=\frac{x_{0}}{t_{0}}\left[1+\frac{a t}{2 t_{0}}\right]^{-1}, \quad f=\frac{c_{*} x_{0}^{2}}{t_{0}^{2}}\left(1+\frac{a \tau}{\rho_{0} x_{0}}\right)^{-1}, \\
\frac{x(t)}{x_{0}}=\frac{2}{a} \ln \left(1+\frac{a t}{2 t_{0}}\right), \\
\frac{x(H)}{x_{0}}=\frac{1}{a} \int_{H_{*}}^{H}\left[2 c W-\frac{\gamma-1}{2 \gamma} H\left(\frac{d W}{d H}\right)^{2}\right] \frac{H^{-\gamma /(\gamma-1)}}{W} d H, \\
\frac{t(H)}{t_{0}}=\frac{1}{a} \int_{H_{*}}^{H}\left[2 c W-\frac{\gamma-1}{2 \gamma} H\left(\frac{d W}{d H}\right)^{2}\right] \frac{H^{-\gamma /(\gamma-1)}}{W^{3 / 2}} d H,
\end{gathered}
$$

where

$$
W=-2 \gamma c H+2 H^{\gamma /(\gamma-1)} .
$$




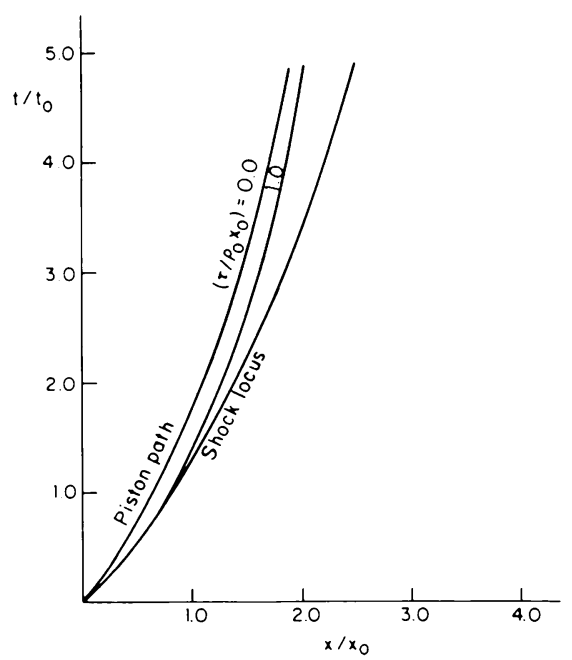

FIG. 4. Piston path, particle trajectory, and shock locus with $\alpha=-0.5, a=1, \gamma=1.4, b_{0}=1$

Fig. 4 shows the piston motion for $\alpha=-0.5, a=1, \gamma=1.4, b_{0}=1$. In this case, the undisturbed density becomes infinite and shock velocity becomes zero in an infinite time and infinite distance, respectively.

(II) Exponential type similarity solutions of the system (7.2)-(7.3). We now choose

$$
\begin{aligned}
& \rho_{*}=\rho_{0} \exp \left(-\frac{a \tau}{\rho_{0} x_{0}}\right), \\
& U=\frac{x_{0}}{t_{0}} \exp \left(\frac{a \tau}{2 \rho_{0} x_{0}}\right), \\
& f=c_{*} \frac{x_{0}^{2}}{t_{0}^{2}} \exp \left(\frac{a \tau}{\rho_{0} x_{0}}\right),
\end{aligned}
$$

where $x_{0}, t_{0}, \rho_{0}$, and $a$ are arbitrary (dimensional) constants, and $c_{*}$ is given by (6.17).

Substitution of (7.34)-(7.36) into (7.6)-(7.8) yields

$$
\begin{aligned}
& W^{(n+1)}(z)=W_{*}^{(n+1)} z^{n+1}, \\
& H^{(n+1)}(z)=H_{*}^{(n+1)} z^{n+1}, \quad n=0,1, \ldots
\end{aligned}
$$

where

$$
z=\frac{t_{0}^{2}}{\rho_{0} x_{0}^{3}} \exp \left(\frac{a \tau}{\rho_{0} x_{0}}\right),
$$


and the constants $W_{*}^{(n+1)}$ and $H_{*}^{(n+1)}, n \geq 0$, are given by

$$
\begin{gathered}
W_{*}^{(n+1)}=\left(\frac{2 \gamma}{\gamma-1}\right)\left(H^{(0)}\right)^{-1}\left[\left(\frac{1}{2} W^{(0)}+c H^{(0)}\right) H_{*}^{(n+1)}-a n H_{*}^{(n)}\right. \\
\left.+T_{*}^{(n)}\left(\left\{W_{*}^{(i)}\right\},\left\{H_{*}^{(i)}\right\}\right)\right], \\
H_{*}^{(n+1)}=\frac{1}{D_{*}}\left[(1-n) a W_{*}^{(n)}-\frac{2 \gamma a n}{\gamma-1}\left(\frac{1}{2} W^{(0)}+c H^{(0)}\right) \frac{H^{(n)}}{H^{(0)}}\right. \\
\left.+S_{*}^{(n)}\left(\left\{W_{*}^{(i)}\right\},\left\{H_{*}^{(i)}\right\}\right)\right],
\end{gathered}
$$

with

$$
\begin{aligned}
T_{*}^{(n)}\left(\left\{W_{*}^{(i)}\right\},\left\{H_{*}^{(i)}\right\}\right)= & \sum_{k=1}^{n}\left(\begin{array}{l}
n \\
k
\end{array}\right)\left[\left(\frac{1}{2} W_{*}^{(k)}+c H_{*}^{(k)}\right) H_{*}^{(n+1-k)}\right. \\
\left.-\frac{\gamma-1}{2 \gamma} H_{*}^{(k)} W_{*}^{(n+1-k)}\right], & \\
S_{*}^{(n)}\left(\left\{W_{*}^{(i)}\right\},\left\{H_{*}^{(i)}\right\}\right)= & \sum_{k=1}^{n}\left(\begin{array}{l}
n \\
k
\end{array}\right)\left[\left(\frac{1}{2} W_{*}^{(k)}+c H_{*}^{(k)}\right) W_{*}^{(n+1-k)}-2 c W_{*}^{(k)} H_{*}^{(n+1-k)}\right] \\
& +\frac{2 \gamma}{\gamma-1}\left(H^{(0)}\right)^{-1}\left(\frac{1}{2} W^{(0)}+c H^{(0)}\right) T_{*}^{(n)}\left(\left\{W_{*}^{(i)}\right\},\left\{H_{*}^{(i)}\right\}\right), \\
D_{*}= & 2 c W^{(0)}-\frac{2 \gamma}{\gamma-1}\left(\frac{1}{2} W^{(0)}+c H^{(0)}\right)^{2}\left(H^{(0)}\right)^{-1}, \\
c= & \frac{1}{2}\left(\frac{2}{\gamma+1}\right)^{(\gamma+1) / \gamma} .
\end{aligned}
$$

Now we may rewrite the series solution (7.4)-(7.5) as

$$
\begin{gathered}
W=\sum_{j=0}^{\infty} W_{*}^{(j)} \frac{\sigma^{j}}{j !}, \\
H=\sum_{j=0}^{\infty} H_{*}^{(j)} \frac{\sigma^{j}}{j !},
\end{gathered}
$$

where $W_{*}^{(j)}$ and $H_{*}^{(j)}, j \geq 0$, are given by (7.10)-(7.11) and (7.39)-(7.40), and

$$
\sigma=\frac{t_{0}^{2}}{\rho_{0} x_{0}^{3}} \exp \left(\frac{a \tau}{\rho_{0} x_{0}}\right) s .
$$

We note that the series solution (7.41)-(7.42) with (7.10)-(7.11) and (7.39)-(7.40) is the solution of the ODE

$$
\left(-a \sigma+\frac{1}{2} W+c H\right) \frac{d H}{d \sigma}-\frac{\gamma-1}{2 \gamma} H \frac{d W}{d \sigma}=0,
$$




$$
\left(-a \sigma+\frac{1}{2} W+c H\right) \frac{d W}{d \sigma}-2 c W \frac{d H}{d \sigma}+a W=0
$$

with boundary conditions (7.10)-(7.11) at the shock. The ODE (7.44)-(7.45) may be obtained from Eqs. (7.2)-(7.3) by using the similarity variable $\sigma$ defined in (7.43). We note that these ODE cannot be solved in a closed form.

The parametric representation of the shock may be found to be

$$
\begin{aligned}
& \frac{x}{x_{0}}=\frac{1}{a}\left[\exp \left(\frac{a \tau}{\rho_{0} x_{0}}\right)-1\right], \\
& \frac{t}{t_{0}}=\frac{2}{a}\left[\exp \left(\frac{a \tau}{2 \rho_{0} x_{0}}\right)-1\right] .
\end{aligned}
$$

The shock trajectory, undisturbed density, and the shock velocity, respectively, are

$$
\begin{gathered}
\frac{x}{x_{0}}=\frac{1}{a}\left[\left(1+\frac{a t}{2 t_{0}}\right)^{2}-1\right], \\
\rho_{*}=\rho_{0}\left(1+\frac{a x}{x_{0}}\right)^{-1}, \\
U=\frac{x_{0}}{t_{0}}\left(1+\frac{a t}{2 t_{0}}\right) .
\end{gathered}
$$

The functions $x, t$, and the piston path are, respectively, given by

$$
\begin{aligned}
\frac{x(H, \tau)}{x_{0}}= & \frac{\exp \left(a \tau / \rho_{0} x_{0}\right)}{a} \int_{H_{*}}^{H}\left[\frac{\gamma-1}{2 \gamma} H\left(\frac{d W}{d H}\right)^{2}-2 c W\right] \frac{H^{-\gamma /(\gamma-1)}}{W} d H \\
& +\frac{1}{a}\left[\exp \left(\frac{a \tau}{\rho_{0} x_{0}}\right)-1\right] \\
\frac{t(H, \tau)}{t_{0}}= & \frac{\exp \left(a \tau / 2 \rho_{0} x_{0}\right)}{a} \int_{H_{*}}^{H}\left[\frac{\gamma-1}{2 \gamma} H\left(\frac{d W}{d H}\right)^{2}-2 c W\right] \frac{H^{-\gamma /(\gamma-1)}}{W^{3 / 2}} d H \\
& +\frac{2}{a}\left[\exp \left(\frac{a \tau}{2 \rho_{0} x_{0}}\right)-1\right] ; \\
\frac{x(H)}{x_{0}}= & \frac{1}{a} \int_{H_{*}}^{H}\left[\frac{\gamma-1}{2 \gamma} H\left(\frac{d W}{d H}\right)^{2}-2 c W\right] \frac{H^{-\gamma /(\gamma-1)}}{W} d H \\
\frac{t(H)}{t_{0}}= & \frac{1}{a} \int_{H_{*}}^{H}\left[\frac{\gamma-1}{2 \gamma} H\left(\frac{d W}{d H}\right)^{2}-2 c W\right] \frac{H^{-\gamma /(\gamma-1)}}{W^{3 / 2}} d H
\end{aligned}
$$

where $H_{*} \leq H<\infty$ if $a=1$, and $1<H \leq H_{*}$ if $a=-1$.

The above solution involves three arbitrary constants $\rho_{0}, x_{0}$, and $t_{0}$. With $a=1$ $(-1)$ the undisturbed density decays to zero in an infinite distance while the shock velocity grows to infinity in an infinite time (the undisturbed density grows to infinity in a finite distance $x_{0}$ and the shock velocity vanishes in finite time $t=2 t_{0}$ ). 
8. Solutions of the system (3.10)-(3.11) with characteristic fronts. Both power-law and exponential similarity forms of the solutions of (3.10)-(3.11) exist also when $b_{0}=\gamma$, the case excluded in Sec. 4. In this special case, Eqs. (4.22)-(4.23) become

$$
\begin{aligned}
& W^{(0)}=1, \\
& H^{(0)}=1,
\end{aligned}
$$

which, in turn, imply that the line $s=0$ is a characteristic, not a shock. This is because the change in pressure, $\left(p-p_{0}\right)$ and velocity $u$ just behind the line $s=0$ are zero (see Eqs. (4.3)). We also find that $c_{*}=1$ in (4.21).

To derive power-law and exponential similarity solutions headed by a characteristic front, we seek a solution of (4.1)-(4.2) in the form (4.7)-(4.8), where the zeroth-order solution is now given by (8.1)-(8.2). We choose the same arbitrary functions $f$ and $\phi$, etc., for power-law similarity solutions as in Sec. 4 .

Power-law similarity solutions of the system (4.1)-(4.2) with characteristic fronts. Substituting (4.7)-(4.8) into Eqs. (4.1)-(4.2), equating the coefficients of $s^{j}, j \geq 0$, to zero and using (4.16)-(4.20), we get

$$
\begin{aligned}
W^{(j)}(z) & =W_{*}^{(j)} z^{j(\alpha+1) / \alpha}, \\
H^{(j)}(z) & =H_{*}^{(j)} z^{j(\alpha+1) / \alpha},
\end{aligned}
$$

with $j=1,2, \ldots$, where

$$
W_{*}^{(1)}=\frac{a \gamma(\alpha+2)}{\gamma+1}, \quad H_{*}^{(1)}=\frac{\gamma-1}{\gamma} W_{*}^{(1)},
$$

and

$$
\begin{aligned}
& W_{*}^{(i)}=-\beta_{0}^{-1}\left[\beta_{1} H_{*}^{(i)}+T_{*}^{(i)}\left(\left\{W_{*}^{(n)}\right\},\left\{H_{*}^{(n)}\right\}\right)\right], \\
& H_{*}^{(i)}=\left[\frac{\beta_{1}}{\beta_{0}}+\frac{\gamma}{\gamma-1}\right]^{-1}\left[(\alpha-(i-1)(\alpha+1)) a W_{*}^{(i-1)}+S_{*}^{(i-1)}\left(\left\{W_{*}^{(n)}\right\},\left\{H_{*}^{(n)}\right\}\right)\right. \\
& \left.+\beta_{0}^{-1} T_{*}^{(i)}\left(\left\{W_{*}^{(n)}\right\} m\left\{H_{*}^{(n)}\right\}\right)\right], \\
& i=2,3, \ldots,
\end{aligned}
$$

with

$$
\begin{aligned}
& \beta_{0}=-\frac{\gamma-1}{\gamma}\left[(\alpha-(\alpha+1) i) a+\left(2+\frac{i}{\gamma}\right) W_{*}^{(1)}\right] \\
& \beta_{1}=(\alpha+1) a i+\left(\frac{\gamma-1}{\gamma}-i\right) W_{*}^{(1)}, \\
& S_{*}^{(i-1)}\left(\left\{W_{*}^{(n)}\right\},\left\{H_{*}^{(n)}\right\}\right)=\sum_{k=1}^{i-1}\left(\begin{array}{c}
i-1 \\
k
\end{array}\right) W_{*}^{(k)} W_{*}^{(i-k)}, \\
& T_{*}^{(i)}\left(\left\{W_{*}^{(n)}\right\},\left\{H_{*}^{(n)}\right\}\right)=\sum_{k=2}^{i-1}\left(\begin{array}{c}
i \\
k
\end{array}\right)\left[\frac{\gamma-1}{\gamma}\left(H_{*}^{(k)}-W_{*}^{(k)}\right) W_{*}^{(i+1-k)}-W_{*}^{(k)} H_{*}^{(i+1-k)}\right],
\end{aligned}
$$


and

$$
z=\frac{1}{p_{0} t_{0}}\left(1+\frac{a \tau}{\rho_{0} x_{0}}\right)^{-(\alpha+1)} .
$$

Using (8.1)-(8.4), the series solution (4.7)-(4.8) may be rewritten as

$$
\begin{aligned}
& W=\sum_{j=0}^{\infty} W_{*}^{(j)} \frac{\sigma^{j}}{j !}, \\
& H=\sum_{j=0}^{\infty} H_{*}^{(j)} \frac{\sigma^{j}}{j !},
\end{aligned}
$$

where

$$
\sigma=\frac{s}{p_{0} t_{0}}\left(1+\frac{a \tau}{\rho_{0} x_{0}}\right)^{-(\alpha+1)} .
$$

The series solution $(8.8)-(8.9),(8.1)-(8.2),(8.6)-(8.7)$ is also the solution of ODE (4.34)-(4.35) with initial conditions

$$
W=P=1 \quad \text { at } \sigma=0 .
$$

The solution (8.8)-(8.9), (8.1)-(8.2), (8.6)-(8.7) involves four arbitrary (dimensional) constants: $x_{0}, t_{0}, p_{0}$ (or $\left.\rho_{0}\right)$, and $\alpha$. The possibility of solutions with characteristic front in the case $b_{0}=\gamma$ was indicated by Ustinov [2]. Sachdev and Reddy [1] also found the first few terms in the local series solution of the ODE (4.34)-(4.35). Again, we note that this series solution $(8.6)-(8.7)$ can be summed up only when $\alpha=-1,-1 / 2$.

For $\alpha=-1$, the solution has the following form:

$$
\begin{aligned}
& u=\phi\left(H^{i /(\gamma-1)}-1\right), \quad \phi=\frac{p_{0} t_{0}}{\rho_{0} x_{0}}\left(1+\frac{a \tau}{\rho_{0} x_{0}}\right)^{-1}, \\
& f(\tau)=\frac{p_{0}^{1 / \gamma}}{\rho_{0}}\left(1+\frac{a \tau}{\rho_{0} x_{0}}\right)^{-2}, \quad \rho_{*}=\rho_{0}\left(1-\frac{a x}{x_{0}}\right)^{-2}, \\
& \frac{x(t)}{x_{0}}=\frac{1}{a}\left[1-\exp \left(-\frac{a t}{t_{0}}\right)\right] \text {. } \\
& \frac{x(H, \tau)}{x_{0}}=\frac{\left(1+a \tau / \rho_{0} x_{0}\right)^{-1}}{(\gamma-1) a}\left[\int_{1}^{H}\left(1-H^{(\gamma+1) /(\gamma-1)}\right) H^{-\gamma^{\prime} /(\gamma-1)} d H\right] \\
& +\frac{1}{a}\left[1-\left(1+\frac{a \tau}{\rho_{0} x_{0}}\right)^{-1}\right] \text {. } \\
& \frac{t(H, \tau)}{t_{0}}=\frac{\gamma}{a(\gamma-1)} \int_{1}^{H}\left(1-H^{(;+1) /(;-1)}\right) \frac{H^{-i /(;-1)}}{\left(H^{i /(;-1)}-1\right)} d H \\
& +\frac{1}{a} \ln \left(1+\frac{a \tau}{\rho_{0} x_{0}}\right) \text {, }
\end{aligned}
$$

where $H \geq 1$ for $a=-1$, and $H \leq 1$ for $a=1$. 
For $\alpha=-1 / 2$, the solution may be found to be

$$
\begin{gathered}
u=\phi G^{1 / 2}, \quad \phi=\frac{p_{0} t_{0}}{\rho_{0} x_{0}}\left(1+\frac{a \tau}{\rho_{0} x_{0}}\right)^{-1 / 2}, \\
f=\frac{p_{0}^{1 / \gamma}}{\rho_{0}}\left(1+\frac{a \tau}{\rho_{0} x_{0}}\right)^{-1}, \quad \rho_{*}=\rho_{0} \exp \left(\frac{a x}{x_{0}}\right), \\
\frac{x(t)}{x_{0}}=\frac{2}{a} \ln \left(1+\frac{a t}{t_{0}}\right), \\
\frac{x(H, \tau)}{x_{0}}=\frac{2}{a \gamma} \int_{1}^{H}\left[\frac{\gamma}{\gamma-1}-\frac{\gamma-1}{\gamma} H\left(\frac{d W}{d H}\right)^{2}\right] H^{-\gamma /(\gamma-1)} d H \\
\frac{t(H, \tau)}{t_{0}}=\frac{2}{a}\left(1+\frac{a \tau}{\rho_{0} x_{0}}\right)^{1 / 2} \int_{1}^{H}\left[\frac{a \tau}{\rho_{0} x_{0}}\right), \\
+\frac{2}{a}\left[\left(1+\frac{a \tau}{\rho_{0} x_{0}}\right)^{1 / 2}-1\right],
\end{gathered}
$$

where

$$
W=G^{1 / 2}+1, \quad G=\frac{2 \gamma}{\gamma-1}(1-\gamma H)+2 \gamma H^{\gamma /(\gamma-1)} .
$$

It follows from (4.44) that $x$ and $t$ are positive in the neighborhood of the characteristic front provided $-2>\alpha>0$ (see also [1]).

9. Conclusions. In the present paper, we have exploited the differential forms of the conservation laws of plane gas-dynamic equations to introduce new coordinate systems $\tau$ and $s$, level lines of which give the particle path and shock trajectory, respectively. The undisturbed conditions and the Rankine-Hugoniot conditions then determine the shock trajectory. The solution behind the shock is obtained by solving the transformed system of PDE. Putting $\tau=0$ in the solution determines the piston motion which generates the shock flow. As detailed in the introduction, the present study extends the earlier works of Sachdev and Reddy [1] and Ustinov [2,3] in several important ways.

It is pertinent to comment upon the nature of solutions, expressed generally in series form. For cases leading to similarity forms, the series solutions are identified as those governed by the corresponding nonlinear ODE; the convergence of the series is therefore proved by checking with the numerical solutions of ODE. In general, the convergence of the series must be shown by computing the series for each set of parameters. The solutions with shocks of arbitrary strength, dealt with in Secs. 4 and 5 , involve constants $x_{0}, t_{0}, \rho_{0}, p_{0}$, and/or $\alpha$, and describe piston-driven shocks into inhomogeneous media which have algebraic form of the undisturbed density. These solutions are characterized by the nondimensional parameters $a(=1$ or -1$)$ and $\alpha$. For example, for the solutions according to (A1) in Sec. 4, the velocity of the shock vanishes in infinite time if $a=1$ and $0>\alpha \geq-1$ and in finite time 
equal to $\left|t_{0} / a(\alpha+1)\right|$ if $-1>\alpha>-\infty$, as it propagates into a medium whose density becomes infinite in infinite distance if $0>\alpha \geq-1 / 2$ and in finite distance $\left|x_{0} / a(2 \alpha+1)\right|$ if $-1 / 2>\alpha>-\infty$. For $a=-1$, the undisturbed density goes to zero at finite distance $\left|x_{0} / 1(2 \alpha+1)\right|$ if $0>\alpha>-1 / 2$ and at infinity if $-1 / 2 \geq \alpha>-\infty$; in this case, the shock velocity grows to become infinite in finite time $\left|t_{0} / a(\alpha+1)\right|$ if $0>\alpha>-1$ and in infinite time if $-1 \geq \alpha>-\infty$. Similar results hold for the solutions according to (A2) in Sec. 5 where $\alpha$ is replaced by $\alpha / 2$.

Turning our attention to the cases of strong shocks in Secs. 6 and 7, we find that the solutions there involve $x_{0}, t_{0}, \rho_{0}$, and/or $\alpha$ (one less than for shocks of finite strength). The density distribution in the undisturbed media and the shock trajectory are the same as for the corresponding cases of arbitrary shock strength, while the flow between the shock and piston trajectories changes. Ustinov's solution for the impulsive piston motion, which is a direct problem, is recovered using the method of series solution proposed in this paper.

The corresponding gas-flows which have a characteristic front rather than a shock are treated in Sec. 8. These solutions can be found following formulation (A1) only if $0>\alpha>-2$. This class of solutions does not seem feasible if we consider formulation (A2). The structure of the solutions and the ambient conditions ahead of the characteristic is similar to those for shocks of finite strength. These solutions are considered a priori, since they cannot be obtained as limiting cases directly from solutions describing shocks of finite strength, as shock strength tends to zero. The solutions here involve $x_{0}, t_{0}, p_{0}$ (or $\rho_{0}$ ), and/or $\alpha$.

The large class of exact solutions for one-dimensional gas-dynamic flows with shocks, reported in this paper, considerably extends the family of known exact solutions.

\section{REFERENCES}

[1] P. L. Sachdev and A. Venkataswamy Reddy, Some exact solutions describing unsteady plane gas flows with shocks, Quart. Appl. Math. 40, 249-272 (1982)

[2] M. D. Ustinov, Ideal gas flow behind a finite-amplitude shock wave, Izv. Akad. Nauk. SSSR Mekh. Zhidk. Gaza 2 no. 1, 88-90 (1967)

[3] M. D. Ustinov, Some one-dimensional unsteady adiabatic gas flows with plane symmetry, Izv. Akad. Nauk SSSR Mekh. Zhidk. Gaza 21 no. 5, 758-765 (1987)

[4] J. A. Steketee, Unsteady rectilinear flow's of a non-homentropic gas, Acta Astronaut. 6, 413-434 (1979)

[5] J. A. Steketee, Homogeneous solutions of the Lagrangian equations of motion (Notes on the unsteady rectilinear motion of a perfect gas III), Report LR-258. Dept. of Aerospace Engineering, Delft University of Technology, Delft, 1977. 